\title{
Large scale magnetogenesis from a non-equilibrium phase transition in the radiation dominated era
}

\author{
D. Boyanovsky ${ }^{(a, b)}$ 团 H. J. de Vega ${ }^{(b, a)}$ 团 and M. Simionato $(a)$ \\ (a) Department of Physics and Astronomy, University of Pittsburgh, Pittsburgh, Pennsylvania 15260, USA \\ (b) LPTHE, Université Pierre et Marie Curie (Paris VI) et Denis Diderot (Paris VII), \\ Tour 16, 1er. étage, 4, Place Jussieu, 75252 Paris, Cedex 05, France
}

(Dated: November 1, 2018)

\begin{abstract}
We study the generation of large scale primordial magnetic fields by a cosmological phase transition during the radiation dominated era. The setting is a theory of $N$ charged scalar fields coupled to an abelian gauge field, that undergoes a phase transition at a critical temperature much larger than the electroweak scale. The dynamics after the transition features two distinct stages: a spinodal regime dominated by linear long-wavelength instabilities, and a scaling stage in which the non-linearities and backreaction of the scalar fields are dominant. This second stage describes the growth of horizon sized domains. We implement a recently introduced formulation to obtain the spectrum of magnetic fields that includes the dissipative effects of the plasma. We find that large scale magnetogenesis is very efficient during the scaling regime. The ratio between the energy density on scales larger than $L$ and that in the background radiation $r(L, T)=\rho_{B}(L, T) / \rho_{c m b}(T)$ is $r(L, T) \sim 10^{-34}$ at the Electroweak scale and $r(L, T) \sim 10^{-14}$ at the QCD scale for $L \sim 1 \mathrm{Mpc}$. The resulting spectrum is insensitive to the magnetic diffusion length. We conjecture that a similar mechanism could be operative after the QCD chiral phase transition.
\end{abstract}

\section{Contents}

I. Introduction 1

II. Magnetic fields in Friedmann-Robertson-Walker cosmology

III. Phase transitions in radiation dominated cosmology

A. Kinematics 5

B. Scalar fields dvnamics 7

1. Spinodal stage 10

2. Scaling stage 13

C. Gauge field dvnamics 14

IV. Magnetic field spectrum 16

A. Magnetogenesis during the spinodal stage 18

B. Magnetogenesis from the scaling regime 20

C. Discussion 22

V. Conclusions 23

References 24

\section{INTRODUCTION}

A variety of astrophysical observations including Zeeman splitting, synchrotron emission, Faraday rotation measurements (RM) combined with pulsar dispersion measurements (DM) and polarization measurements suggest the presence of large scale magnetic fields 1, 2, 3, 4, 5, 6. The strength of typical galactic magnetic fields is measured to be $\sim \mu G[2,[3,4,[6]$ and they are correlated on very large scales up to galactic or even larger reaching to scales

\footnotetext{
*Electronic address: boyan@pitt.edu

${ }^{\dagger}$ Electronic address: devega@lpthe.jussieu.fr

${ }^{\ddagger}$ Electronic address: mis6@pitt.edu
} 
of cluster of galaxies $\sim 1 \mathrm{Mpc}$ 2, 3, 4, 5. The origin of these large scale magnetic fields is still a subject of much discussion and controversy. It is currently agreed that a variety of dynamo mechanisms are efficient in amplifying seed magnetic fields with typical growth rates $\Gamma \sim \mathrm{Gyr}^{-1}$ over time scales $\sim 10-12 \mathrm{Gyr}$ (for a thorough discussion of the mechanisms and models see [4]). The ratio of the energy density of the seed magnetic fields on scales larger than $L$ (today) to that in the cosmic background radiation, $r(L)=\rho_{B}(L) / \rho_{c m b}$ must be $r(L \sim 1 \mathrm{Mpc}) \geq 10^{-34}$ for a dynamo mechanism to amplify it to the observed value, or $r(L \sim 1 \mathrm{Mpc}) \geq 10^{-8}$ for the seed to be amplified solely by the gravitational collapse of a protogalaxy $[5]$.

There are also different proposals to explain the origin of the initial seed. Astrophysical batteries rely on gradients of the charge density concentration and pressure and their efficiency in producing seeds of the necessary amplitude is still very much discussed [2, 4]. Primordial magnetic fields that could be the seeds for dynamo amplification can be generated at different stages in the history of the early Universe, in particular during inflation, preheating and or phase transitions [3, 4, 5]. Primordial (hyper) magnetic fields may have important consequences in electroweak baryogenesis [7], Big Bang nucleosynthesis (see[3]), the polarization of the CMB [8] via the same physical processes as Faraday rotation, and structure formation 3, 5, 9], thus sparking an intense program to study the origin and consequences of the generation of magnetic fields in the early Universe 10 - 24.

A reliable estimate of the amplitude and correlations of seed magnetic fields must include the dissipative properties of the plasma, in particular the conductivity [11, 13, 14]. In ref. 25] we have introduced a formulation that allows to compute the generation of magnetic fields from processes strongly out of equilibrium. This formulation, which is based on the exact set of Schwinger-Dyson equations for the transverse photon propagator is manifestly gauge invariant and is general for any matter fields and any cosmological background (conformally related to Minkowski space-time). In the case in which strongly out of equilibrium effects arise from long-wavelength fluctuations, such as during phase transitions, this formulation allows to separate the contribution of the hard degrees of freedom which are in local thermodynamic equilibrium from that of the soft degrees of freedom that fall out of LTE (local thermal equilibrium) during the phase transition and whose dynamics is strongly out of equilibrium. This separation of degrees of freedom leads to a consistent incorporation of the dissipative effects via the conductivity (for details see[25]). In that reference a study of magnetogenesis in Minkowski space-time during a supercooled phase transitions was presented and the results highlighted the main aspects of the generation of magnetic and electric fields in these situations.

The goals of this article: In this article we study the generation of large scale (hyper) magnetic fields by a cosmological phase transition during a radiation dominated era by implementing the formulation introduced in ref.[25]. The setting is a theory of $N$ charged scalar fields coupled to an abelian gauge field (hypercharge). We consider the situation when this theory undergoes a phase transition after the reheating stage and before either the Electroweak or the QCD phase transition, since we expect that these transitions will lead to new physical phenomena. The non-perturbative dynamics out of equilibrium is studied in the limit of a large number $N$ of (hyper) charged fields and to leading order in the gauge coupling. The non-equilibrium dynamics of the charged scalar sector features two distinct stages. The first one describes the early and intermediate time regime and is dominated by the spinodal instabilities which are the hallmark of the process of phase separation and domain formation and growth. This stage describes the dynamics between the time at which the phase transition takes place and that at which non-linearities become important via the backreaction. The second stage corresponds to a scaling regime which describes the slower non-equilibrium evolution of Goldstone bosons and the process of phase ordering [26] and growth of horizon-sized domains. This scaling regime is akin to the solution found in the classical evolution of scalar field models with broken continuous symmetries after the phase transition that form the basis for models of structure formation based on topological defects [27, 28].

The solution of the scalar field dynamics [26] is the input in the expression for the spectrum of the magnetic field obtained in 25] to obtain the amplitude of the primordial seed generated during both stages.

We find that scaling stage is the most important for the generation of large scale magnetic fields. Large scale magnetic fields are generated via loop effects from the dynamics of modes that are at the scale of the horizon or smaller. The resulting spectrum is rather insensitive to the diffusion length scale which is much smaller than the horizon during the radiation dominated era. The ratio of the magnetic energy density on scales larger than $L$ (today) to the energy density in the background radiation $r(L, \eta)=\rho_{B}(L, \eta) / \rho_{c m b}(\eta)$ is summarized in a compact formula [eq. (4.34)]. For $L \sim 1 \mathrm{Mpc}$ (today) we find $r(L, \eta) \sim 10^{-34}$ at the Electroweak scale and $r(L, \eta) \sim 10^{-14}$ at the QCD scale, suggesting the possibility that these primordial seeds could be amplified by dynamo mechanisms to the values of the magnetic fields consistent with the observed ones on these scales.

In section II we introduce the model, in section III we summarize the dynamics in the different stages after the phase transition and discuss the dynamics of gauge fields including the dissipative effects of the plasma. In section IV we compute the spectrum of the primordial magnetic field generated during the different stages and assess the regime of validity of the approximations invoked. Our results and conclusions are summarized in section V. 


\section{MAGNETIC FIELDS IN FRIEDMANN-ROBERTSON-WALKER COSMOLOGY}

The cosmological setting in which we are primarily interested corresponds to a symmetry breaking phase transition in a radiation dominated Universe. Such phase transition is in principle different from the electroweak one ${ }^{1}$ and presumably occurs at a much higher energy scale, such as the GUT scale $\sim 10^{15} \mathrm{Gev}$ but is assumed to be described by a particle physics model that includes many fields with (hyper)-charge either fermionic or bosonic. We will not attempt to study a particular gauge theory phenomenologically motivated by some GUT scenario, but will focus our study on a generic scalar field model in which the scalar fields carry an abelian (hyper)charge. The simplest realization of such model is scalar electrodynamics with $N$ charged scalar fields $\phi_{r}, r=1, \ldots, N$ and one neutral scalar field $\psi$ whose expectation value is the order parameter associated with the phase transition.

This model is inspired by the $O(4)$ linear sigma model which is the low energy effective theory of QCD that describes chiral symmetry breaking and the dynamics of pions [29, 30]. In this low energy effective theory the neutral field associated with $\psi \sim<\bar{q} q>$ acquires an expectation value while the three pion fields $\pi^{ \pm}, \pi^{0}$ are the (quasi)-Goldstone modes associated with chiral symmetry breaking. The charged pions couple minimally to the electromagnetic field [29] and obviously chiral symmetry breaking (a nonzero expectation for the neutral field) preserves the gauge symmetry. We argue later that the model [see eq.(2.1)] can describe magnetogenesis during the QCD phase transition. The mechanisms of magnetogenesis discussed in the present article is therefore akin to the photoproduction during the nonequilibrium chiral phase transition 31 .

The neutral field is not coupled to the gauge field and its acquiring an expectation value does not break the $U(1)$ gauged symmetry. This guarantees that the abelian gauge symmetry identified with either hypercharge or electromagnetism is not spontaneously broken to describe the correct low energy sector with unbroken $U(1)_{E M}$. We will take the neutral and the $N$ complex (charged) fields to form a scalar multiplet under an $O(2 N+1)$ isospin symmetry. As the neutral field acquires an expectation value this isospin symmetry is spontaneously broken to $O(2 N)$. The explicit breaking of the $O(2 N+1)$ symmetry induced by the electromagnetic coupling further reduce this symmetry to $S U(N) \times U(1)$. If the neutral field acquires a non vanishing expectation value, the isospin symmetry breaking does not affect the masslessness of the photon (it will obtain a Debye screening mass from medium effects).

The action that describes this theory in a general cosmological background is given by

$$
S=\int d^{4} x \sqrt{-g}\left[g^{\mu \nu}\left(\frac{1}{2} \partial_{\mu} \psi \partial_{\nu} \psi+\mathcal{D}_{\mu} \phi^{*} \mathcal{D}_{\nu} \phi\right)+\mu^{2}\left(\frac{\psi^{2}}{2}+\phi^{*} \phi\right)-\frac{\lambda}{4 N}\left(\frac{\psi^{2}}{2}+\phi^{*} \phi\right)^{2}-\frac{1}{4} \mathcal{F}_{\mu \nu} \mathcal{F}_{\alpha \beta} g^{\mu \alpha} g^{\nu \beta}\right]
$$

where

$$
\mathcal{D}_{\mu}=\partial_{\mu}-i e \mathcal{A}_{\mu} \quad \text { and } \quad \mathcal{F}_{\mu \nu}=\partial_{\mu} \mathcal{A}_{\nu}-\partial_{\nu} \mathcal{A}_{\mu}
$$

and

$$
\phi^{\dagger} \phi=\sum_{r=1}^{N} \phi_{r}^{\dagger} \phi_{r}, \quad D_{\mu} \phi^{\dagger} D^{\mu} \phi=\sum_{r=1}^{N}\left(\partial_{\mu}+i e A_{\mu}\right) \phi_{r}^{\dagger}\left(\partial^{\mu}-i e A^{\mu}\right) \phi_{r} .
$$

Furthermore, anticipating a non-perturbative treatment of the non-equilibrium dynamics of the scalar sector in a large $N$ expansion, we have rescaled the quartic coupling in such a way as to display the contributions in terms of powers of $1 / N$, keeping $\lambda$ fixed in the large $N$ limit.

A Friedmann-Robertson-Walker line element

$$
d s^{2}=d t^{2}-a^{2}(t) d \vec{x}^{2},
$$

is conformally related to a Minkowski line element by introducing the conformal time $\eta$ and scale factor $C(\eta)$ as

$$
\eta=\int \frac{d t}{a(t)} ; C(\eta)=a(t(\eta))
$$

In terms of these the line element and metric are given by

$$
d s^{2}=C^{2}(\eta)\left(d \eta^{2}-d \vec{x}^{2}\right) ; g_{\mu \nu}=C^{2}(\eta) \eta_{\mu \nu}
$$

\footnotetext{
${ }^{1}$ If the electroweak phase transition is weakly first order, nucleation will be almost indistinguishable from spinodal decomposition and the phenomena studied here may be of relevance.
} 
where $\eta_{\mu \nu}=\operatorname{diag}(1,-1,-1,-1)$ is the Minkowski metric. Introducing the conformal fields

$$
A_{\mu}(\eta, \vec{x})=\mathcal{A}(t(\eta), \vec{x}) ; \Phi_{r}(\eta, \vec{x})=C(\eta) \phi_{r}(t(\eta), \vec{x}), \quad 1 \leq r \leq N ; \Psi(\eta, \vec{x})=C(\eta) \psi(t(\eta), \vec{x})
$$

and in terms of the conformal time, the action now reads

$$
S=\int d \eta d^{3} x\left[\eta^{\mu \nu}\left(\frac{1}{2} \partial_{\mu} \Psi \partial_{\nu} \Psi+D_{\mu} \Phi^{*} D_{\nu} \Phi\right)-M^{2}(\eta)\left(\frac{\Psi^{2}}{2}+\Phi^{*} \Phi\right)-\frac{\lambda}{4 N}\left(\frac{\Psi^{2}}{2}+\Phi^{*} \Phi\right)^{2}-\frac{1}{4} F_{\mu \nu} F_{\alpha \beta} \eta^{\mu \nu} \eta^{\alpha \beta}\right]
$$

with

$$
M^{2}(\eta)=-\mu^{2} C^{2}(\eta)-\frac{C^{\prime \prime}(\eta)}{C(\eta)} \quad, \quad D_{\mu}=\partial_{\mu}-i e A_{\mu} \quad ; \quad F_{\mu \nu}=\partial_{\mu} A_{\nu}-\partial_{\nu} A_{\mu},
$$

and the primes refer to derivatives with respect to conformal time. Obviously the conformal rescaling of the metric and fields turned the action into that of a charged scalar field interacting with a gauge field in flat Minkowski spacetime, but the scalar field acquires a time dependent mass term ${ }^{2}$. In particular, in the absence of electromagnetic coupling, the equations of motion for the gauge field $A_{\mu}$ are those of a free field in flat space time. This is the statement that gauge fields are conformally coupled to gravity and no generation of electromagnetic fields can occur from gravitational expansion alone without coupling to other fields or breaking the conformal invariance of the gauge sector. The generation of electromagnetic fields must arise from a coupling to other fields that are not conformally coupled to gravity, or by adding extra terms in the Lagrangian that would break the conformal invariance of the gauge fields 11.

The conformal electromagnetic fields $\overrightarrow{\mathcal{E}}, \overrightarrow{\mathcal{B}}$ are related to the physical $\vec{E}, \vec{B}$ fields by the conformal rescaling

$$
\vec{E}=\frac{\overrightarrow{\mathcal{E}}}{C^{2}(\eta)} ; \vec{B}=\frac{\overrightarrow{\mathcal{B}}}{C^{2}(\eta)},
$$

corresponding to fields of scaling dimension two. A gauge invariant formulation leads to the following Lagrangian density (for details see 25, 32])

$$
\begin{aligned}
\mathcal{L}= & \frac{1}{2} \partial_{\mu} \Psi \partial^{\mu} \Psi+\partial_{\mu} \Phi^{\dagger} \partial^{\mu} \Phi+\frac{1}{2} \partial_{\mu} \vec{A}_{T} \cdot \partial^{\mu} \vec{A}_{T}+\frac{1}{2}\left(\nabla A_{0}\right)^{2}-M^{2}(\eta)\left(\frac{1}{2} \Psi^{2}+\Phi^{\dagger} \Phi\right)-\frac{\lambda}{4 N}\left(\frac{1}{2} \Psi^{2}+\Phi^{\dagger} \Phi\right)^{2} \\
& -i e \vec{A}_{T} \cdot\left(\Phi^{\dagger} \nabla \Phi-\nabla \Phi^{\dagger} \Phi\right)-e^{2}\left(\vec{A}_{T}^{2}-A_{0}^{2}\right) \Phi^{\dagger} \Phi-i e A_{0}\left(\dot{\Phi}^{\dagger} \Phi-\Phi^{\dagger} \dot{\Phi}\right)
\end{aligned}
$$

where $\Phi$ is a gauge invariant local field which is non-locally related to the original fields, and $\vec{A}_{T}$ is the transverse component of the vector field $\left(\vec{\nabla} \cdot \vec{A}_{T}=0\right)$ and $A_{0}$ is a non-propagating field as befits a Lagrange multiplier, its dynamics is completely determined by that of the charge density [25, 32].

The main point of this discussion is that the framework to obtain the power spectrum of the generated magnetic field presented below is fully gauge invariant.

The theory described by the Lagrangian eq.(2.6) above bears some similarity to the scalar-gauge field theory that describes semilocal strings 33, 34, 35, however there are important differences between the model studied here and that studied in refs. [33, 34, 35]: i) we assume that symmetry breaking occurs along the neutral direction thus the charged scalar field does not acquire an expectation value, whereas in the semilocal theory of refs. 33, 34, 35. the charged fields acquire an expectation value, and the gauge symmetry is spontaneously broken. ii) We study the dynamics beginning from an initial state in LTE above the critical temperature, follow the dynamics through the phase transition and compute systematically to lowest order in $\alpha_{e m}$ the non-equilibrium spectrum of magnetic fields generated by the process of phase separation. The goal of the studies in ref. [34] are very different focusing on the rate of production of semilocal strings. The initial state studied in these references places the scalar field at the minimum of the (classical) potential and the phases are distributed at random, with particular initial conditions on the gauge fields, namely conditions corresponding to zero temperature, broken symmetry states. Furthermore the dynamics in ref. 34 is studied in flat space time with an ad hoc dissipative term for the scalar field.

In contrast, we study the full quantum dynamics beginning from a state of LTE above $T_{c}$ evolving the quantum Heisenberg equations of motion and calculate the magnetic field consistently to lowest order in $\alpha$. Thus while the theory studied here and that proposed in refs. 33, 34, 35] bear a resemblance, they describe very different physics and we study a different set of phenomena.

\footnotetext{
${ }^{2}$ Here we neglect the effect of the conformal anomaly [20]
} 


\section{PHASE TRANSITIONS IN RADIATION DOMINATED COSMOLOGY}

\section{A. Kinematics}

We consider a phase transition corresponding to the dynamics of small field models where the scalar field has vanishing expectation value but with a symmetry breaking potential, namely at the top of the potential hill.

In a radiation dominated cosmology, the initial state is that of local thermodynamic equilibrium at an initial temperature $T>>T_{c}$. Using finite temperature field theory in an expanding background geometry, it is shown [26] that the effective time dependent mass term depends on the effective time dependent temperature $T(t)=T / a(t)$ which reflects the cooling from the cosmological expansion (see below). Hence at a given time the temperature equals the critical and the phase transition occurs. Field modes with wavectors much larger than the symmetry breaking scale $\mu$ will remain in LTE and will not be affected by the symmetry breaking dynamics 36 .

We normalize the scaling factor $C(\eta)$ at the reheating time $\eta=\eta_{R}$ in such a way that $C\left(\eta_{R}\right)=1$ then the explicit expression for $C(\eta)$ reads

$$
C(\eta)=H_{R} \eta
$$

where $H_{R}$ is the Hubble constant at the reheating time, $H_{R}=\eta_{R}^{-1}$.

We can relate $H_{R}$ to the reheating temperature and the Planck mass $G^{-\frac{1}{2}}$ through the equation

$$
\rho=\frac{\pi^{2} g_{*}}{30} T_{R}^{4}
$$

and the Einstein-Friedman equation

$$
H_{R}=\left(\frac{8}{3} \pi G \rho\right)^{1 / 2}=\frac{T_{R}^{2}}{M_{*}}
$$

where $g^{*}$ is the effective number of degrees of freedom at the reheating temperature and we introduced the scale $M_{*}$ of the order of the Planck mass

$$
M_{*}=\frac{3 \sqrt{5}}{2 \pi^{\frac{3}{2}}} \frac{1}{\sqrt{g_{*} G}} .
$$

In radiation dominated epoch the time-dependence of the mass term (2.7) is given by the expression

$$
-M^{2}(\eta)=\mu^{2} H_{R}^{2} \eta^{2}=\tilde{\mu}^{4} \eta^{2},
$$

where we see the emergence of a new mass scale

$$
\tilde{\mu}=\sqrt{\mu H_{R}} .
$$

This scale will play an important role in the following discussion and in the comparison with results obtained in Minkowski space-time [25]. There is a last scale which plays a relevant role, the horizon scale $r_{H}(\eta)$ which is fixed by the evolution on the time of the Hubble constant:

$$
r_{H}(\eta)=\frac{1}{H(\eta)}=C(\eta) \eta=H_{R} \eta^{2} .
$$

Modes with physical wavelength $\lambda_{\text {phys }}=\frac{2 \pi}{k_{\text {phys }}}$ inside the horizon

$$
\lambda_{\text {phys }}(\eta) \sim k_{\text {phys }}^{-1}(\eta)<r_{H}(\eta)
$$

are causally connected, modes outside the horizon are causally disconnected.

The relaxation rate of hard modes of the charged fields is given by [37]

$$
\Gamma(\eta) \sim \alpha T(\eta) \ln \frac{1}{\alpha}
$$

where the effective temperature varies with time as

$$
T(\eta)=\frac{T_{R}}{C(\eta)}
$$


and the expansion rate given by

$$
H(\eta)=\frac{T^{2}(\eta)}{M_{*}}
$$

Therefore,

$$
\frac{\Gamma(\eta)}{H(\eta)} \sim \frac{10^{16}}{T(\eta)[\mathrm{Gev}]} .
$$

Thus hard modes are in thermal equilibrium for $T(\eta) \leq 10^{15} \mathrm{Gev}$.

In particular, modes with $k \sim T_{R}$ are the hard modes that give the leading contribution to the conductivity in the high temperture limit [38, 39]. Modes with $k<\mu$ will manifest the long-wavelength spinodal instabilities and their dynamics will be strongly out of equilibrium 36, 40, 41, 42]. Their amplitude becomes non-perturbatively large 36, 40, 41, 42] and will be responsible for the non-equilibrium generation of the primordial magnetic field [25].

Using eqs. (3.1) and (3.10) we can write the conformal time as

$$
\eta=\frac{T_{R}}{H_{R} T(\eta)} .
$$

As it will become clear below an important cosmological quantity is the product

$$
k \eta=\frac{k}{C(\eta)} \mathcal{C}(\eta) \eta=\frac{k_{p h y s}(\eta)}{T(\eta)} T(\eta) r_{H}(\eta)=\frac{2 \pi}{L T_{R}} \frac{M_{*}}{T(\eta)} .
$$

The ratio

$$
\frac{k_{p h y s}(\eta)}{T(\eta)}=\frac{2 \pi}{L T_{R}}
$$

is a kinematical invariant. Its value today is determined by the scale $L$ which will be typically chosen to correspond to a galactic scale or the scale of galaxy clusters, and the temperature of the CMB. It is given by,

$$
L T_{R}=3.7 \times 10^{25}\left(\frac{L}{\mathrm{Mpc}}\right) .
$$

Therefore,

$$
k \eta \sim 10^{-9} \frac{T_{E W}}{T(\eta)}\left(\frac{\mathrm{Mpc}}{L}\right)=\left\{\begin{array}{l}
10^{-22} \text { for } T(\eta)=T_{R} \sim 10^{15} \mathrm{Gev} \\
10^{-9} \text { for the EW transition } \\
10^{-6} \text { for the QCD transition }
\end{array}\right.
$$

for $L \sim 1 \mathrm{Mpc}$. Thus, during the regime of interest in this article, $k \eta \ll 1$ for scales of galaxy clusters. A noteworthy aspect of eq.(3.17) is that the wavelengths corresponding to the scale of galaxies or clusters today were well outside the horizon during the radiation dominated era when the electroweak and QCD phase transitions occurred.

Another important quantity is the ratio of the wavevector $k$ of the primordial magnetic field to the conductivity.

As it will be discussed below, the physical conductivity is given by

$$
\sigma(\eta)=\frac{\mathcal{C} N(\eta) T(\eta)}{\alpha \ln \frac{1}{\alpha N(\eta)}},
$$

where $\mathcal{C}$ is a constant of $\mathcal{O}(1), N(\eta)$ is the number of ultrarelativistic charged species, and we have neglected the (logarithmic) dependence on the energy scale in the running coupling constant. For this discussion we will neglect the time dependence of $N(\eta)$ assuming that the number of charged ultrarelativistic species remains constant (this assumption can be relaxed without qualitative modifications of the main argument). Under this assumption

$$
\sigma(\eta)=\frac{\sigma_{R}}{C(\eta)},
$$

with $\sigma_{R}$ being the comoving conductivity determined at the time of reheating

$$
\sigma_{R}=\frac{\mathcal{C} N T_{R}}{\alpha \ln \frac{1}{\alpha N}} .
$$


Thus the ratio,

$$
\frac{k_{p h y s}(\eta)}{\sigma(\eta)} \sim \frac{2 \pi \alpha}{N L T_{R}} \sim 10^{-27}\left(\frac{\mathrm{Mpc}}{L}\right)
$$

neglecting logarithmic corrections.

Furthermore,

$$
\sigma(\eta) r_{H}(\eta) \sim \frac{M_{*}}{\alpha T(\eta)} \sim\left\{\begin{array}{l}
10^{5} \text { for } T(\eta)=T_{R} \sim 10^{15} \mathrm{Gev} \\
10^{18} \text { for the EW phase transition } \\
10^{21} \text { for the QCD phase transition }
\end{array}\right.
$$

where we have neglected logarithmic corrections. Therefore $\sigma_{R} \eta \gg 1$ throughout the radiation dominated era considered in this article. The regime $\sigma_{R} \eta \gg 1 ; k^{2} \eta / \sigma_{R} \ll 1$ is dominated by the (slow) hydrodynamic relaxation of the magnetic field.

Another relevant estimate involves the (comoving) diffusion length $\xi_{\text {diff }}(\eta)=\sqrt{\eta / \sigma_{R}}$

$$
\frac{\xi_{\text {diff }}(\eta)}{\eta} \sim \sqrt{\frac{\alpha T(\eta)}{M_{*}}} \sim\left\{\begin{array}{l}
10^{-3} \text { for } T(\eta)=T_{R} \sim 10^{15} \mathrm{Gev} \\
10^{-9} \text { for the EW phase transition } \\
10^{-10} \text { for the QCD phase transition }
\end{array}\right.
$$

where again we have neglected logarithmic terms. Therefore the diffusion length is much smaller than the Hubble radius during the radiation dominated era. Finally, combining eqs.(3.23) and (3.17) we find

$$
10^{-25} \leq k \xi_{\text {diff }}(\eta) \leq 10^{-16}
$$

between reheating and the time of the QCD phase transition.

The contribution from the hard modes of both the charged scalar and gauge fields which remain in local thermodynamic equilibrium lead to an effective mass for the scalar field. This thermal mass is obtained from the longwavelength limit of the scalar field self-energy and includes the hard thermal loop contributions from the gauge and scalar fields [43, 44]. This thermal mass is given by

$$
m_{T}^{2}=\frac{T_{R}^{2}}{24}\left(\lambda+3 e^{2}\right) .
$$

Finally, another important quantity is the Debye screening length that determines the scale at which long-range forces are screened by the polarizability of the medium. In an ultrarelativistic plasma, the comoving Debye screening length is given by 43,44$]$

$$
\xi_{D} \sim \frac{1}{e T_{R}}
$$

the ratio of the Debye screening length to the Hubble radius is given by

$$
\frac{\xi_{D}}{d_{H}} \sim \frac{1}{e} \frac{T(\eta)}{M_{*}}
$$

Hence $\xi_{D} \ll d_{H}(\eta)$ for $T(\eta) \leq 10^{16} \mathrm{Gev}$, thus long range forces are screened over very short distances. The formation of long-wavelength domains with typical size of the order of the Hubble radius [26] leading to strong charge and current fluctuations that will seed magnetic fields, will not be hindered by long-range forces, which are effectively screened over sub-horizon distances.

Magnetic field generation via charge asymmetries during a period in which electromagnetism was spontaneously broken was previously studied by Dolgov and Silk [45] who argued that long-range forces would be screened by the Higgs mechanism. This is different from the situation studied in this article, where the $U(1)$ symmetry associated with electromagnetism (rather hypercharge) is not spontaneously broken. Long range forces are screened by the plasma, a situation not considered in [45].

\section{B. Scalar fields dynamics}

For completeness and to highlight the aspects of the non-equilibrium dynamics most relevant to the generation of magnetic fields, we summarize the main features of scalar field dynamics. For further details the reader is referred 
to $40,41,42$. In what follows we will neglect the backreaction of the gauge fields on the dynamics of the scalar fields. The rationale for this is that the main non-equilibrium processes that lead to magnetogenesis will be non-perturbative in the scalar sector and result from the instabilities associated with the phase transition. The contribution from the gauge fields, in the form of self-energies for the scalar fields, do not feature the instabilities associated with the phase transition and will, furthermore, be suppressed at least by one power of $\alpha$ the (hyper) electromagnetic coupling constant as compared to the scalar self-interaction.

As described above, the non-equilibrium evolution of long-wavelength modes begins with the spinodal instabilities which result in an exponential growth of the amplitudes for long-wavelength fluctuations. When the non-linearity becomes of the same order as the tree-level terms in the equations of motion, the back reaction of these fluctuations shuts-off the instabilities [40, 41, 42]. Therefore a non-perturbative treatment of the dynamics is required. The large $N$ limit of the scalar sector allows a systematic non-perturbative treatment of the dynamics which is renormalizable and maintains the conservation laws 40,41$]$.

We will therefore study the dynamics in leading order in the large $N$ limit that already reveals the important non-equilibrium features of the evolution.

\section{Radiative corrections:}

The contribution from the gauge fields to the equations of motion of the long-wavelength modes of the scalar fields arise through self-energy corrections. To lowest order in $\alpha$ these are dominated by the hard modes of the gauge fields with momenta $\sim T$ (hard thermal loops) which lead to a contribution to the thermal mass given by $e T / \sqrt{8}$ [3], 44]. Thus the lowest order radiative corrections had already been accounted for in the thermal mass eq. 3.25.

The non-equilibrium effects in the gauge contribution of the scalar self-energy will arise from polarization loops in the photon propagator. This in turn will induce non-equilibrium radiative corrections to the self-energy of the scalar fields of the order $\mathcal{O}\left(\alpha^{2}\right)$. These small contributions can be safely neglected in this context. Thus, to this order the radiative corrections to the scalar field from the gauge field propagator in the scalar self-energy are accounted for in the thermal mass.

Hence the dynamics of the scalar field is studied along the same lines as presented in refs. 40, 41, 42] but the only difference is in the initial conditions in the modes that reflect the thermal mass in LTE.

Since symmetry breaking is chosen along the direction of the neutral field $\Psi$ we write

$$
\Psi(\vec{x}, \eta)=\sqrt{N} \varphi(\eta)+\chi(\vec{x}, \eta) \quad ; \quad\langle\chi(\vec{x}, \eta)\rangle=0
$$

where the expectation value is taken in the time evolved density matrix or initial state. The leading order in the large $N$ limit is obtained either by introducing an auxiliary field and establishing the saddle point or equivalently by the factorizations 40,41$]$

$$
\begin{aligned}
& \left(\Phi^{\dagger} \Phi\right)^{2} \rightarrow 2\left\langle\Phi^{\dagger} \Phi\right\rangle \Phi^{\dagger} \Phi \\
& \chi \Phi^{\dagger} \Phi \rightarrow \chi\left\langle\Phi^{\dagger} \Phi\right\rangle .
\end{aligned}
$$

This factorization that yields the leading contribution in the large $N$ limit makes the Lagrangian for the scalar fields quadratic (in the absence of the gauge coupling) at the expense of a self-consistent condition: thus charged fields $\phi$ acquire a self-consistent time dependent mass.

The dynamics is determined by the Heisenberg equations of motion of the neutral field $\Psi$ and the charged fields $\Phi$ [40, 41, 42]. We will consider that at the onset of the radiation dominated era, the system is in the symmetric high temperature phase in local thermal equilibrium with a vanishing expectation value for the scalar fields. Consequently, the initial conditions are $\langle\Psi(\vec{x}, 0)\rangle=\sqrt{N} \varphi(0)=0,<\dot{\Psi}(\vec{x}, 0)>=\sqrt{N} \dot{\varphi}(0)=0,<\Phi_{r}(0, \vec{x})>=0,<\dot{\Phi}{ }_{r}(0, \vec{x})>=$ 0 .

In the absence of explicit symmetry breaking perturbations the expectation value of the scalar field will remain zero throughout the evolution, thus $\varphi \equiv 0$.

It is convenient to introduce the mode expansion of the charged fields

$$
\Phi_{r}(\eta, \vec{x})=\int \frac{d^{3} k}{\sqrt{2(2 \pi)^{3}}}\left[a_{r}(\vec{k}) f_{k}(\eta) e^{i \vec{k} \cdot \vec{x}}+b_{r}^{\dagger}(\vec{k}) f_{k}^{*}(\eta) e^{-i \vec{k} \cdot \vec{x}}\right] \quad, \quad r=1, \ldots, N
$$

with $<a_{r}(\vec{k})>=<b_{r}(\vec{k})>=0$.

In leading order in the large $N$ limit, the Heisenberg equations of motion for the charged fields translate into the following equations of motion for the mode functions and the expectation value of the neutral field for $\eta>$ $\eta_{R}$, 40, 41, 42.

$$
\left[\frac{d^{2}}{d \eta^{2}}+k^{2}-M^{2}(\eta)+\frac{\lambda}{2} \varphi^{2}(\eta)+\frac{\lambda}{2 N}\left\langle\Phi^{\dagger} \Phi\right\rangle\right] f_{k}(\eta)=0
$$




$$
\left[\frac{d^{2}}{d \eta^{2}}-M^{2}(\eta)+\frac{\lambda}{2} \varphi^{2}(\eta)+\frac{\lambda}{2 N}\left\langle\Phi^{\dagger} \Phi\right\rangle\right] \varphi(\eta)=0 .
$$

Obviously, the initial conditions $\varphi(0)=0, \dot{\varphi}(0)=0$ imply that $\varphi(\eta)=0$ for all times. That is, $\varphi(\eta)=0$ is a fixed point of the dynamics.

We must now append initial conditions for the mode functions $f_{k}(\eta)$. The initial conditions on the mode functions $f_{k}(\eta)$ depend on the value of the wavevector $k$ as compared to the horizon scale $H_{R}^{-1}$ at the reheating time:

- $\mathbf{k}>\mathbf{H}_{\mathbf{R}}$ : for fluctuations inside the horizon, we may assume thermal quasi-particle boundary conditions at the reheating temperature:

$$
f_{k}\left(\eta_{R}\right)=\frac{1}{\sqrt{W_{k}}} \quad ; \quad f_{k}^{\prime}\left(\eta_{R}\right)=-i W_{k} f_{k}\left(\eta_{R}\right), \quad W_{k}=\sqrt{k^{2}+m_{T}^{2}},
$$

where the frequencies $W_{k}$ are quasi-particle frequencies with thermal mass $m_{T}^{2}$ given by eq.(3.25) at the reheating temperature,

$$
W_{k}=\sqrt{k^{2}+m_{T}^{2}} .
$$

For these modes the assumption of local thermodynamic equilibrium is well motivated and we have

$$
\left\langle a_{r}^{\dagger}(\vec{k}) a_{s}(\vec{k})\right\rangle=\left\langle b_{r}^{\dagger}(k) b_{s}(k)\right\rangle=\delta_{r s} n_{k} \quad ; \quad n_{k}=\frac{1}{e^{\frac{W_{k}}{T}}-1}
$$

- $\mathbf{k}<\mathbf{H}_{\mathbf{R}}$ : for superhorizon fluctuations, which are causally disconnected at the reheating time, we cannot assume a thermalized distribution. The correct distribution has to be derived by following the dynamics from the inflationary stage, when the fluctuations were well inside the horizon. While a complete discussion of the initial conditions is left to a forthcoming article, the case under consideration we will see that the dependence on the initial conditions is rather weak and only during the initial stages of the phase transition. For the later stages, dominated by the scaling solution described below, the dynamics is universal and does not depend on the initial conditions. We will simply assume that both $W_{k}$ and $n_{k}$ have a finite non-zero limit as $k \rightarrow 0$ namely the only important quantities for the dynamics of long-wavelength fluctuations are

$$
\lim _{k \rightarrow 0} W_{k}=W_{0}, \quad 0<W_{0}<\infty, \quad \lim _{k \rightarrow 0} n_{k}=n_{0}, \quad 0<n_{0}<\infty .
$$

With this choice of the initial state we find the backreaction term to be given by

$$
\frac{\lambda}{2 N}\left\langle\Phi^{\dagger} \Phi\right\rangle=\frac{\lambda}{4} \int \frac{d^{3} k}{(2 \pi)^{3}}\left|f_{k}(\eta)\right|^{2}\left[1+2 n_{k}\right]
$$

This expectation value is ultraviolet divergent, it features quadratic and logarithmic divergences in terms of an upper momentum cutoff. The quadratic divergence and part of the logarithmic divergence (the one proportional to the mass term) are absorbed in a renormalization of the mass term $\mu^{2} \rightarrow \mu_{R}^{2}$ and the remainder logarithmic divergence is absorbed into a renormalization of the scalar coupling $\lambda \rightarrow \lambda_{R}$. While these aspects are not relevant for the discussion here, they are mentioned for completeness, the reader is referred to [26] for details.

After renormalization the self-consistent field $\frac{\lambda}{2 N}\left\langle\Phi^{\dagger} \Phi\right\rangle$ is subtracted twice, and is given by (for details see [26] and references therein)

$$
\begin{aligned}
\frac{\lambda}{2 N}\left\langle\Phi^{\dagger} \Phi\right\rangle & =\lambda_{R}[J(\eta)+I(\eta)] \quad, \quad \lambda_{R} J(\eta)=\frac{\lambda_{R}}{4 \pi^{2}} \int_{0}^{\infty} q^{2} d q\left|f_{q}(\eta)\right|^{2} n_{q} \\
\lambda_{R} I(\eta) & =\frac{\lambda_{R}}{8 \pi^{2}} \int_{0}^{\infty} q^{2} d q\left\{\left|f_{q}(\eta)\right|^{2}-\frac{1}{q}+\frac{\Theta\left(q-K^{2}\right)}{2 q^{3}}\left[-\mu_{R}^{2}+\frac{\lambda}{2 N}\left\langle\Phi^{\dagger} \Phi\right\rangle\right]\right\} .
\end{aligned}
$$

and the mass and coupling are replaced by their renormalized counterparts $\mu_{R}^{2} ; \lambda_{R}$ respectively. Here $K$ is an arbitrary renormalization scale. In order to avoid cluttering of notation we now drop the subscript $R$ for renormalized quantities, in what follows $\mu ; \lambda$ stand for the renormalized quantities.

The finite temperature term $J(\eta)$ has contributions from short wavelengths for which the mode functions are of the form $f_{q}(\eta) \sim e^{i q \eta} / \sqrt{q}$ and contributions from long wavelengths. The contribution from short wavelengths is the 
same as that in equilibrium in Minkowski space time and determines the hard-thermal loop [43, 44] contribution to the self-energy given by 26$]$

$$
J_{H T L}=\frac{T_{R}^{2}}{24}
$$

where we have used that the short wavelength modes are in thermal equilibrium at the reheating temperature $T_{R}$. This hard thermal loop contribution has been self-consistently accounted for in the thermal mass of the scalar field eq.(3.25).

It is convenient to separate the hard thermal loop component eq.(3.36) from eq.(3.35) and define

$$
\lambda \Sigma(\eta)=\frac{\lambda}{8 \pi^{2}} \int_{0}^{\infty} q^{2} d q\left\{\left|f_{q}(\eta)\right|^{2}\left(1+2 n_{q}\right)-\frac{1}{q}\left[1+\frac{2}{e^{\frac{q}{T_{R}}}-1}\right]+\frac{\Theta\left(q-K^{2}\right)}{2 q^{3}}\left[-\mu^{2}+\lambda \Sigma(\eta)\right]\right\} .
$$

After renormalization and in terms of dimensionless quantities, the non-equilibrium dynamics of the charged scalar fields is completely determined by the following equations of motion [26, 40, 41, 42],

$$
\left[\frac{d^{2}}{d \eta^{2}}+\mathcal{M}^{2}(\eta)+q^{2}+\lambda \Sigma(\eta)\right] f_{q}(\eta)=0 \quad ; \quad f_{q}\left(\eta_{R}\right)=\frac{1}{\sqrt{W_{q}}} \quad ; \quad f^{\prime}{ }_{q}\left(\eta_{R}\right)=-i W_{q} f_{q}\left(\eta_{R}\right)
$$

with $W_{q}$ given by eq. (3.5) and the effective, (conformal) time dependent mass is given by

$$
\begin{aligned}
\mathcal{M}^{2}(\eta) & =C^{2}(\eta) \mu^{2}\left[\frac{T_{R}^{2}}{C^{2}(\eta) T_{c}^{2}}-1\right] \\
T_{c}^{2} & =\frac{24 \mu^{2}}{\lambda+3 e^{2}} .
\end{aligned}
$$

Where we have used $\varphi=0$ as a fixed point of the dynamics.

The time dependent mass term $\mathcal{M}^{2}(\eta)$ includes the high temperature corrections and clearly displays the cooling associated with the expansion in the form of a time dependent effective temperature $T_{\text {eff }}(\eta)=T_{R} / C(\eta)$. The phase transition occurs at a time $\eta_{c}$ when $T_{\text {eff }}\left(\eta_{c}\right)=T_{c}$, thus for $\eta>\eta_{c}$ the effective time dependent mass term is $\mathcal{M}^{2}(\eta)=M^{2}(\eta)=-\mu^{2} H_{R}^{2} \eta^{2}=-\tilde{\mu}^{4} \eta^{2}$ as given by equations (3.5)-(3.6).

The full time evolution of mode functions in a radiation dominated cosmology has been studied analytically and numerically in detail in ref. 26, 40]. Here we highlight the most important features which are necessary ingredients to study magnetogenesis. The reader is referred to [26] for a more comprehensive discussion.

The are two main dynamical stages in the evolution:

- Spinodal stage: this is the stage immediately after the phase transition which is dominated by spinodal decomposition and the growth of long-wavelength fluctuations [25]. This stage spans the time scale $\eta_{c} \leq \eta \leq \eta_{n l}$ where the non-linear time scale $\eta_{n l}$ is determined by (see below)

$$
\eta_{n l}^{2}=\frac{\lambda \Sigma\left(\eta_{n l}\right)}{\tilde{\mu}^{4}}
$$

During this stage the back-reaction, determined by the term $\lambda \Sigma(\eta)$, can be neglected and the dynamics is linear.

- Scaling stage: This is a stage in which the non-linearity encoded by the back-reaction term $\lambda \Sigma(\eta)$ are very important and compete with the tree level term in the equations of motion. This stage is described by a scaling solution of the equations of motion for the modes with small wavevectors and describes the non-equilibrium relaxation of long-wavelength fluctuations [26, 27, 28].

\section{Spinodal stage}

After the phase transition but before the non-linear time scale after which the back-reaction becomes important, namely for $\eta_{c} \ll \eta<\eta_{n l}$ the time dependent mass term is given by $\mathcal{M}^{2}=-\tilde{\mu}^{4} \eta^{2}$, and for weak coupling $\lambda \ll 1$ we can neglect the back-reaction $\lambda \Sigma(\eta)$. The equations of motion for the mode functions during this stage are given by

$$
\left[\frac{d^{2}}{d \eta^{2}}+q^{2}-\tilde{\mu}^{4} \eta^{2}\right] f_{q}(\eta)=0 \quad ; \quad q<\tilde{\mu}^{2} \eta
$$


We note that for $T_{c} \sim 10^{15} \mathrm{GeV}$

$$
\tilde{\mu} \eta_{c}=\left(\sqrt{\frac{\lambda+3 e^{2}}{24}} \frac{M_{*}}{T_{c}}\right)^{1 / 2} \sim 10
$$

and therefore for $\eta>\eta_{c}$ we are in the regime $\tilde{\mu} \eta \gg 1$. It is clear that the mode functions $f_{q}(\eta)$ will increase exponentially in the band of unstable wavevectors $q<\tilde{\mu}^{2} \eta$. Eq.3.42 can be solved exactly in terms of Hermite functions [6]

$$
f_{q}(\eta)=b_{q} e^{-\frac{1}{2}(\tilde{\mu} \eta)^{2}} H_{\frac{1}{2}\left(\frac{q^{2}}{\tilde{\mu}^{2}}-1\right)}(\tilde{\mu} \eta)+a_{q} e^{\frac{1}{2}(\tilde{\mu} \eta)^{2}} H_{-\frac{1}{2}\left(\frac{q^{2}}{\tilde{\mu}^{2}}+1\right)}(i \tilde{\mu} \eta)
$$

where the constants $a_{q}$ and $b_{q}$ are fixed by the initial conditions (3.38). For $\tilde{\mu} \eta \gg 1$ we can use the asymptotic behavior of the Hermite functions [6],

$$
H_{\nu}(z) \stackrel{z \gg 1}{=}(2 z)^{\nu}\left[1+\mathcal{O}\left(\frac{1}{z^{2}}\right)\right]
$$

and we find for the mode functions,

$$
f_{q}(\eta) \stackrel{\tilde{\mu}}{\underline{\eta} \gg 1} a_{q} e^{\frac{1}{2}(\tilde{\mu} \eta)^{2}}(\tilde{\mu} \eta)^{-\frac{q^{2}}{\tilde{\mu}^{2}}-1}\left[1+\mathcal{O}\left(\frac{1}{\tilde{\mu}^{2} \eta^{2}}\right]\right]
$$

Since the exponentially damped solution becomes negligible the phases of the mode functions $f_{q}(\eta)$ freeze, namely, they become constant in time and are slowly varying functions of $q$ for long wavelengths.

This is very similar to the situation in Minkowski space-time, where the mode functions however increase as $e^{\mu t}$, i.e. much slower. In any case the soft $(q \rightarrow 0)$ modes are the most amplified at the end of the evolution, therefore, the quantum fluctuations (3.37) are dominated by the lower integration bound $q=0$.

We notice that the freezing of the long-wavelength mode functions will play an important role in the discussion about the magnetic field generation, since it assures the independence of the final result from the initial particle distribution function, except for subleading corrections.

The physics of the phase transition is essentially the same as in Minkowski space-time 25, 40, 41, 42], since the exponential growth of modes in the spinodally unstable band will make the back reaction term $\lambda \Sigma(\eta)$ begin to grow and eventually cancel the term $-\tilde{\mu}^{4} \eta^{2}$ in the equations of motion (for $\eta>>\eta_{c}$ the effective time dependent temperature vanishes).

This will happen at a non-linear time scale defined by [40, 41]

$$
\lambda \Sigma\left(\eta_{n l}\right)=\tilde{\mu}^{4} \eta_{n l}^{2}
$$

Two important aspects are described by $\eta_{n l}$ : i) at this time scale the phase transition is almost complete since $\lambda \Sigma\left(\eta_{n l}\right)=\tilde{\mu}^{4} \eta_{n l}^{2}$ means that $\lambda\left\langle\Phi^{\dagger} \Phi\right\rangle / 2 N=\tilde{\mu}^{4} \eta_{n l}^{2}$, namely the mean square root fluctuations in the scalar field probe the manifold of minima of the potential.

ii) At $\eta \sim \eta_{n l}$ the mean square root fluctuations of the field are of order $M^{2}\left(\eta_{n l}\right) / \lambda$ probing the vacuum manifold, and the non-linearities become very important. The back reaction $\lambda \Sigma\left(\eta_{n l}\right)$ becomes comparable to $M^{2}\left(\eta_{n l}\right)$ and the instabilities shut-off. Thus for $\eta_{c}<\eta<\eta_{n l}$ the dynamics is described by the linear spinodal instabilities while for $\eta>\eta_{n l}$ a full non-linear treatment of the evolution is required. As it will be discussed below this later stage is described by the emergence of a scaling solution.

For $\eta_{n l}>\eta \gg \tilde{\mu}^{-1}$ the asymptotic form (3.45) for the mode functions apply and we find for the the quantum fluctuations (3.37) which dominated by the lower integration bound $q=0$,

$$
\lambda \Sigma\left(\eta_{n l}\right)=\lambda\left(1+2 n_{0}\right) \frac{\tilde{\mu}^{2}\left|a_{0}\right|^{2}}{32 \pi^{\frac{5}{2}}} \frac{e^{\tilde{\mu}^{2} \eta_{n l}^{2}}}{\tilde{\mu} \eta_{n l}\left[\ln \left(\tilde{\mu} \eta_{n l}\right)\right]^{\frac{3}{2}}}\left[1+\mathcal{O}\left(\frac{1}{\tilde{\mu} \eta_{n l}}\right)\right]
$$

This leads to the following estimate for the spinodal time for weak coupling $\lambda$

$$
\eta_{n l}^{2}=\frac{1}{\tilde{\mu}^{2}}\left[\ln \left(\frac{32 \pi^{\frac{5}{2}}}{\lambda\left(1+2 n_{0}\right)\left|a_{0}\right|^{2} \tilde{\mu}}\right)+\frac{3}{2} \ln \ln \left(\frac{32 \pi^{\frac{5}{2}}}{\lambda\left(1+2 n_{0}\right)\left|a_{0}\right|^{2} \tilde{\mu}}\right)+\mathcal{O}\left(\ln \ln \ln \frac{1}{\lambda}\right)\right] .
$$


The important point is that the dependence on boundary conditions and the initial distribution is solely logarithmic, thus we may expect out predictions to be very robust with respect to changes of the initial conditions. In particular, the scale factor at this non-linear time scale is given by

$$
C\left(\eta_{n l}\right)=\frac{T_{R}}{\sqrt{M_{*} T_{c}}}\left(\frac{24}{\lambda+3 e^{2}}\right)^{\frac{1}{4}} \sqrt{\ln \frac{1}{\lambda}}\left[1+\mathcal{O}\left(\frac{1}{\ln \frac{1}{\lambda}}\right)\right],
$$

where we have used eqs. (3.3) and (3.40).

The amplitude of the long-wavelength modes at the non-linear time, roughly speaking at the end of the phase transition is approximately

$$
\left|f_{q}\left(\eta_{n l}\right)\right|=\sqrt{\frac{32 \pi^{\frac{5}{2}}}{\lambda\left(1+2 n_{0}\right) \tilde{\mu}}}\left[\ln \frac{32 \pi^{\frac{5}{2}}}{\lambda\left(1+2 n_{0}\right)}\right]^{\frac{1}{4}-\frac{q^{2}}{2 \tilde{\mu}^{2}}}
$$

As we will discuss in detail below this non-perturbative scale will ultimately determine the strength of the magnetic fields generated during the phase transition.

During the intermediate time regime the equal times correlation function is approximately

$$
\left\langle\Phi_{q, a}^{\dagger}(\eta) \Phi_{q, b}(\eta)\right\rangle=\delta_{a, b}\left|a_{q}\right|^{2} e^{\tilde{\mu}^{2} \eta^{2}} e^{-\left(\frac{q^{2}}{\tilde{\mu}^{2}}+1\right) \ln [\tilde{\mu} \eta]} .
$$

and its Fourier transform for long wavelenghts is of the form

$$
\left\langle\Phi_{a}(\vec{x}, \eta) \Phi_{b}(\overrightarrow{0}, \eta)\right\rangle=\delta_{a, b}\left|a_{0}\right|^{2} \frac{e^{\tilde{\mu}^{2} \eta^{2}}}{\tilde{\mu} \eta} \tilde{\mu}^{3}\left[\frac{\pi}{\ln \tilde{\mu} \eta}\right]^{\frac{3}{2}} e^{-\frac{\vec{x}^{2}}{\xi^{2}(\eta)}}
$$

which determines the time dependent correlation length of the scalar field,

$$
\xi(\eta)=\frac{2}{\tilde{\mu}} \sqrt{\ln \tilde{\mu} \eta}=2 \sqrt{\frac{\ln \left(\sqrt{\mu H_{R}} \eta\right)}{\mu H_{R}}} .
$$

This expression is valid in the intermediate time regime $\eta_{c}<\eta<\eta_{n l}$ during which the non-equilibrium dynamics is dominated by the spinodal instabilities. The detailed analysis of the dynamics in refs. 40, 41, 42] and the discussion of the main features presented above can be summarized as follows:

- At intermediate times $\tilde{\mu}^{-1} \ll \eta \leq \eta_{n l} \sim \tilde{\mu}^{-1} \sqrt{\ln 1 / \lambda}$ the mode functions grow exponentially for modes in the spinodally unstable band $q<M(\eta)$. The phase of these mode functions freezes, namely, becomes independent of time and slowly varying with momentum.

- At a time scale determined by the spinodal time the back-reaction shuts off the instabilities and the phase transition is almost complete. This can be understood from the following: the backreaction becomes comparable with the tree-level term (for $\eta>\eta_{R}$ ) when $\frac{\lambda}{2 N}\left\langle\Phi^{\dagger} \Phi\right\rangle \approx \tilde{\mu}^{4} \eta^{2}$. This relation determines that the mean square root fluctuation of the scalar field probes the minima of the tree level potential.

- During the spinodal stage the correlation length of the scalar field grows in time and is given by eq.(3.53). This is interpreted as the formation of correlated domains that grow in time, and is the hallmark of the process of phase separation and ordering. This correlation length will be important in the analysis of the correlation of magnetic fields later.

- The large fluctuations associated with the growth of spinodally unstable modes of the charged fields will lead to current fluctuations which in turn will lead to the generation magnetic fields. Thus the most important aspect of the non-equilibrium dynamics of the charged fields during the phase transition is that large fluctuations of the charged fields associated with the spinodal instabilities will lead to the generation of magnetic fields. Since the modes with longer wavelength are the most unstable the magnetic field generated through the process of phase separation will be of long wavelength. Furthermore we expect that the magnetic field generated by these non-equilibrium processes will be correlated on length scales of the same order as that of the charged field above. 


\section{Scaling stage}

A remarkable result of the evolution in the asymptotic regime (when the effective temperature has vanished) found in ref. [26] is that there is a very precise cancellation between the tree level term $-\mu^{2} C^{2}(\eta)$ and the back reaction $\lambda \Sigma(\eta)$ in the equations of motion (3.38). The self-consistency condition requires that for a radiation dominated cosmology 26$]$

$$
\lambda \Sigma(\eta)-\mu^{2} C^{2}(\eta) \stackrel{\eta \rightarrow \infty}{=}-\frac{15}{4 \eta^{2}}
$$

In this asymptotic regime the solutions of the equations of motion

$$
\left[\frac{d^{2}}{d \eta^{2}}+k^{2}-\frac{15}{4 \eta^{2}}\right] f_{k}(\eta)=0
$$

are given by

$$
f_{k}(\eta)=\sqrt{\eta}\left[A_{k} \frac{J_{2}(k \eta)}{k^{2}}+B_{k} k^{2} N_{2}(k \eta)\right] .
$$

This solution can be written in terms of the scaling variable

$$
x=k \eta
$$

in a more illuminating form

$$
f_{k}(\eta)=A_{k} \eta^{5 / 2} \frac{J_{2}(x)}{x^{2}}+B_{k} \frac{x^{2} N_{2}(x)}{\eta^{3 / 2}},
$$

As discussed in detail in ref. [26], the relevant integrals are dominated by $x \sim 1$, namely by modes with wavelength of the order of the Hubble radius, thus the second contribution proportional to $N_{2}(x)$ can be safely neglected at long times.

For $x \lesssim 1$ in the long time regime we can further approximate $A_{k} \sim A_{0}$ and the asymptotic solution during this stage is of the scaling form

$$
f_{k}(\eta)=A_{0} \eta^{5 / 2} \frac{J_{2}(x)}{x^{2}} .
$$

Since for $x \lesssim 3$ and large time the modes with small wavevector have the largest amplitudes, these dominate the backreaction. The very precise cancellation (3.54) leads to the following sum rule [26]

$$
\frac{\lambda}{8 \pi^{2}} \int k^{2} d k\left|f_{k}(\eta)\right|^{2}\left(1+2 n_{0}\right) \stackrel{\eta \rightarrow \infty}{=} \tilde{\mu}^{4} \eta^{2}
$$

Since for large $\eta$ the integral is dominated by soft modes $k \sim \frac{1}{\eta} \rightarrow 0$ the distribution function can be approximated by $n_{0}$ and the amplitude by $\left|A_{0}\right|^{2}$. The sum rule eq.(3.60) then leads to the identity

$$
\left|A_{0}\right|^{2}\left(1+2 n_{0}\right)=\frac{30 \pi^{3}}{\lambda} \mu^{2} H_{R}^{2}
$$

where we used the integral $[46]$

$$
\int_{0}^{\infty} \frac{d x}{x^{2}} J_{2}^{2}(x)=\frac{4}{15 \pi}
$$

which is dominated by $x \lesssim 3$. This is a remarkable result: the product $\left|A_{0}\right|^{2}\left[1+2 n_{0}\right]$ in the scaling regime does not depend on the initial conditions on the evolution, namely it is universal in the sense that it is independent of the previous history through the phase transition. This is an important result which will play an important role in the power spectrum of the magnetic fields.

An important consequence of this scaling solution is that the equal time two-point correlation function of the scalar field is given by

$$
\left\langle\Phi_{a}(\vec{x}, \eta) \Phi_{b}(\overrightarrow{0}, \eta)\right\rangle=\delta_{a, b} D(z) ; z=\frac{|\vec{x}|}{2 \eta}
$$


which reveals that the correlation length is given by the size of the causal horizon 26]. The dynamical evolution during the scaling stage is precisely determined by the growth of horizon-sized domains [26].

We summarize below the important features of the solutions in the scaling regime that will be used in the computation of the power spectrum of the magnetic fields.

- For $\eta>>\eta_{n l}$ a scaling regime emerges in which the mode functions are given by eq. (3.59) with $x=k \eta$. This scaling solution describes the relaxation of long-wavelength fluctuations of the charged fields. Again the phase of these modes freezes namely is independent of time. This is important because this fact will entail that the retarded self-energy of the transverse photon polarization tensor will give a subleading contribution to the generation of magnetic fields.

- The sum rule eq.(3.60) constrains the product of the amplitude times the occupation of the long wavelength scaling modes to be given by eq.(3.61).

- The scaling solution described above is akin to that found in classical models of formation of topological defects [27, 28]. The scaling regime describes the evolution of long-wavelength fluctuations and the adjustment of the spatial correlation length of the scalar field to the Hubble radius 26.

\section{Gauge field dynamics}

In a high temperature plasma a very important aspect that must be taken into account in the dynamics of gauge fields is the electric conductivity, which leads to dissipative processes. As discussed in [25], the electric conductivity severely hinders magnetogenesis, and also introduces the diffusion length scale which could limit the correlation of the magnetic fields that are generated.

In Minkowski space in equilibrium the conductivity is obtained from the imaginary part of the photon polarization and it is dominated by charged particles of momenta $p \sim T$ in the loop with exchange of photons of momenta $e T<k \ll T$ 38, 39]. A careful analysis including Debye (electric) and dynamical (magnetic) screening via Landau damping leads to the conclusion that the conductivity is given by [38, 39]

$$
\sigma=\frac{\mathcal{C} N T}{\alpha \ln \frac{1}{\alpha N}}
$$

with $N$ the number of charged fields and $\mathcal{C} \sim \mathcal{O}(1)$.

In an expanding cosmology an in particular during phase transitions, a more precise assessment of the contributions and meaning of the conductivity must be provided. As it was discussed in ref. [25], the fluctuations of the charged fields during a phase transition will have very different behavior if the typical wavevector of these modes is of the order of or smaller than the symmetry breaking scale or much larger than this scale.

Short wavelength modes, those with typical wave vectors much larger than the symmetry breaking scale are insensitive to the phase transition and are always in local thermodynamic equilibrium (LTE). For short wavelength modes deep inside the horizon, the mode functions are of the free field type $f_{q}(\eta) \sim e^{i q \eta} / \sqrt{q}$.

Long wavelength modes, those with wavectors of the order of or smaller than the symmetry breaking scale undergo critical slowing down and fall out of equilibrium during the phase transition. These modes become spinodally unstable during the early stages of the transition as summarized above and analyzed in detail in refs. 26, 40, 41, 42].

Thus the contributions from the charged particle fluctuations to the photon polarization must be separated into two very different regimes: a) the hard momenta $p \gg|M(\eta)|$ correspond to charge fluctuations that are always in local thermodynamic equilibrium, b) the soft momenta $p \ll|M(\eta)|$ fall out of equilibrium and undergo long-wavelength spinodal instabilities and enter the scaling regime.

The contribution from hard momenta will lead to a large equilibrium conductivity in the medium, while the contribution to the polarization from soft momenta will contain all the non-equilibrium dynamics that lead to the generation of electromagnetic field fluctuations.

As the instabilities during the phase transition develop, the fluctuations of the charged fields will generate nonequilibrium fluctuations in the long-wavelength components of the electric and magnetic fields with the ensuing generation of long-wavelength magnetic fields. However the large conductivity of the medium will hinder the generation of electromagnetic fluctuations, hence the conductivity must be fully taken into account to assess the spectrum of the magnetic and electric fields generated during the non-equilibrium stage [25].

We are interested in the generation of long wavelength magnetic fields, namely $k<<T$ but also $k<<\alpha^{2} T$, since within the astrophysical application, the wavelength of interest for magnetic fields are of galactic scale, while $T$ corresponds to a wavelength at the peak of the CMB $\sim$ millimeters. Thus the physical situation corresponds to studying the photon polarization tensor for long-wavelength. 
The polarization tensor has local (tadpole) and non-local contributions. The equilibrium contribution to the tadpole $\sim\left\langle\Phi^{\dagger} \Phi\right\rangle$ is dominated by momenta $\sim T_{R}$ leading to the hard-thermal loop result $\left\langle\Phi^{\dagger} \Phi\right\rangle \sim T_{R}^{2}$. This contribution is actually cancelled by the zero frequency-momentum limit of the non-local polarization (bubble diagram). This cancellation in equilibrium is a consequence of the Ward identities and implies the vanishing of the magnetic mass in absence of symmetry breaking 44, 47]. In equilibrium the inverse propagator vanishes in the limit of zero frequency and momentum [44, 47] in absence of symmetry breaking at any temperature.

Out of equilibrium, the tadpole and the non-local polarization (bubble diagram) exactly cancel each other in the longwavelength, longtime limit as shown in ref. 48] [see eq.(6.12) in the reference] indicating the vanishing of the photon mass.

In equilibrium the long-wavelength and low frequency limit $(k, \omega \rightarrow 0)$ of the spatial and temporal Fourier transform of the transverse polarization is given by

$$
\Pi_{T}(k, \omega)=i \omega \sigma
$$

Thus we write for the full transverse polarization for long-wavelength electromagnetic fields

$$
\Pi_{T}\left(\eta, \eta^{\prime}, k\right)=\sigma \frac{d}{d \eta^{\prime}} \delta\left(\eta-\eta^{\prime}\right)+\Pi_{n o n e q}\left(\eta, \eta^{\prime}, k\right)
$$

The first term above includes the contribution from the hard momentum modes $p \sim T_{R}$ in the transverse polarization, while $\Pi_{\text {noneq }}\left(\eta, \eta^{\prime}, k\right)$ is the contribution from the long wavelength modes which are unstable in the spinodal stage and take the scaling form in the scaling regime. Thus in a very well defined sense, the polarization (3.65) describes the effective low energy theory for the transverse photon field.

Our strategy is to obtain the non-equilibrium contribution to the spectrum of electromagnetic fields to lowest order in $\alpha$ but treating the conductivity exactly.

In a cosmological space-time, the temperature scales with the inverse of the conformal factor

$$
T(\eta)=\frac{T_{R}}{C(\eta)}
$$

and therefore the conductivity $\sigma=\sigma(\eta)$ becomes time-dependent. If we are interested in time scales where the number of ultrarelativistic charge carriers does not change significantly, which is the case that we will consider in what follows, then the time evolution of the conductivity is purely kinematic:

$$
\sigma(\eta)=\frac{\sigma_{R}}{C(\eta)} .
$$

An important effect of the conductivity, as discussed in [25], is the introduction of a diffusion scale in the transverse photon propagator. The long-time behavior of the zeroth order propagators for the transverse gauge fields: retarded $(\mathrm{R})$, advanced $(\mathrm{A})$, symmetric $(\mathrm{H})$

$$
\mathcal{D}_{R, A, H}^{i j}\left(\eta, \eta^{\prime}, k\right)=\mathcal{P}^{i j}(\hat{\mathbf{p}}) \mathcal{D}_{R, A, H}\left(\eta, \eta^{\prime}, k\right)
$$

obey [1] (see 25] for details)

$$
\begin{aligned}
& {\left[\frac{d^{2}}{d \eta^{2}}+k^{2}+\sigma(\eta) C(\eta) \frac{d}{d \eta}\right] \mathcal{D}_{R}\left(\eta, \eta^{\prime}, k\right)=\delta\left(\eta-\eta^{\prime}\right) ; \mathcal{D}_{R}\left(\eta, \eta^{\prime}\right)=0 \text { for } \eta<\eta^{\prime}} \\
& {\left[\frac{d^{2}}{d \eta^{2}}+k^{2}+\sigma(\eta) C(\eta) \frac{d}{d \eta}\right] \mathcal{D}_{A}\left(\eta, \eta^{\prime}, k\right)=\delta\left(\eta-\eta^{\prime}\right) ; \mathcal{D}_{A}\left(\eta, \eta^{\prime}\right)=0 \text { for } \eta>\eta^{\prime}} \\
& {\left[\frac{d^{2}}{d \eta^{2}}+k^{2}+\sigma(\eta) C(\eta) \frac{d}{d \eta}\right] \mathcal{D}_{H}\left(\eta, \eta^{\prime}, k\right)=0,}
\end{aligned}
$$

with the transverse projector

$$
\mathcal{P}_{i j}(\hat{\mathbf{p}})=\delta^{i j}-\hat{\mathbf{p}}^{i} \hat{\mathbf{p}}^{j} .
$$

Due to eq. (3.67) the comoving conductivity $\sigma_{R}=\sigma(\eta) C(\eta)$ is an invariant quantity in the regime in which the number of ultrarelativistic charge carriers is constant. The estimate given by eq.(3.22) clearly indicates that during the radiation dominated era between reheating and the QCD phase transition, $\sigma_{R} \eta \gg 1$.

Then for $k \ll \sigma_{R}$ (which is certainly fulfilled since the relevant wavevectors are $k \ll T \ll T / \alpha \sim \sigma_{R}$ ) and $\eta \gg 1 / \sigma_{R}$ we can safely neglect the second order time derivatives in eqs.(3.68), leading to the following equations,

$$
\begin{aligned}
& \mathcal{D}_{R}\left(\eta, \eta^{\prime}, k\right)=\mathcal{D}_{C}\left(\eta, \eta^{\prime} ; k\right) \theta\left(\eta-\eta^{\prime}\right) \quad, \quad \mathcal{D}_{A}\left(\eta, \eta^{\prime}, k\right)=\mathcal{D}_{C}\left(\eta, \eta^{\prime} ; k\right) \theta\left(\eta^{\prime}-\eta\right) \\
& \mathcal{D}_{H}\left(\eta, \eta^{\prime}, k\right)=i \frac{e^{-\frac{k^{2}}{\sigma_{R}}\left(\eta+\eta^{\prime}\right)}}{\sigma_{R}} \quad, \quad \mathcal{D}_{C}\left(\eta, \eta^{\prime} ; k\right)=\frac{e^{-\frac{k^{2}}{\sigma_{R}}\left(\eta-\eta^{\prime}\right)}}{\sigma_{R}} .
\end{aligned}
$$




\section{MAGNETIC FIELD SPECTRUM}

As discussed in detail in reference 25], the quantity of astrophysical relevance is the correlation function

$$
<\hat{B}^{i}(\eta, \vec{x}) \hat{B}^{i}(\eta, \overrightarrow{0})>_{\rho}
$$

where the sum on repeated indices is understood. $B(\eta, \vec{x})$ above is a Heisenberg operator and the expectation value is in the initial density matrix. From this quantity, the spectrum of the magnetic field is obtained in the coincidence limit

$$
S_{B}(\eta, k)=\frac{1}{2} \lim _{\eta^{\prime} \rightarrow \eta} \int d^{3} x<\left\{\hat{B}^{i}(\eta, \vec{x}), \hat{B}^{i}\left(\eta^{\prime}, \overrightarrow{0}\right)\right\}>_{\rho} e^{i \vec{k} \cdot \vec{x}},
$$

where $\{$,$\} denotes the anti commutator. And from S_{B}(\eta, k)$ we can extract the physical magnetic energy density stored on comoving length scales larger than a given $L$

$$
\Delta \rho_{B}(L, \eta)=\frac{1}{2 \pi^{2}} \int_{0}^{\frac{2 \pi}{L}} k^{2} S_{B}(\eta, k) d k .
$$

where we have restored the powers of the scale factor arising from the transformation to conformal time. Denoting by $\Delta \rho_{B}(L, \eta)$ the contribution from the non-equilibrium generation (subtracting the local thermodynamic equilibrium contribution), a quantity of cosmological relevance to assess the relative strength of the generated magnetic field is given by the ratio of the power on scales larger than $L$ to the energy density in the radiation background

$$
r(L, \eta)=\frac{\Delta \rho_{B}(L, \eta)}{\rho_{\gamma}(\eta)}
$$

where

$$
\rho_{\gamma}=\frac{\pi^{2} T_{R}^{4}}{15}
$$

is the comoving energy density in the thermal equilibrium background of photons.

The physical energy densities $\Delta \rho_{B, p h y s}(L, \eta), \rho_{\gamma, p h y s}$ are obtained from the comoving expressions above by rescaling $\rho \rightarrow \rho / C^{4}(\eta)$ as can be seen from the conformal rescaling (2.8). Thus the ratio $r(L, \eta)$ would be a constant in the absence of non-equilibrium generation or dissipative processes. Hence the time dependence of the ratio (4.4) only is solely a consequence of the non-equilibrium generation mechanisms or dissipative processes (such as magnetic diffusion in a conducting plasma) but not through the cosmological expansion.

Using the results obtained in reference 25] that lead to a first principle derivation of the spectrum, we just quote its expression to leading order in $\alpha$, (here and in what follows $S_{B}$ refers solely to the non-equilibrium contribution to the spectrum)

$$
S_{B}(\eta, k)=S_{B}^{I}(\eta, k)+S_{B}^{H}(\eta, k)
$$

with

$$
\begin{aligned}
& S_{B}^{I}(\eta, k)=e^{2} N \int \frac{d^{3} q}{(2 \pi)^{3}} q^{2}\left(1-\cos ^{2} \theta\right)\left[\left(1+n_{q}\right)\left(1+n_{|\vec{q}+\vec{k}|}\right)\left|\int_{\eta_{R}}^{\eta} d \eta_{1} k \mathcal{D}_{C}\left(\eta, \eta_{1}, k\right) f_{q}\left(\eta_{1}\right) f_{|\vec{q}+\vec{k}|}\left(\eta_{1}\right)\right|^{2}+\right. \\
& +\left(1+n_{q}\right) n_{|\vec{q}+\vec{k}|}\left|\int_{\eta_{R}}^{\eta} d \eta_{1} k \mathcal{D}_{C}\left(\eta, \eta_{1}, k\right) f_{q}\left(\eta_{1}\right) f_{|\vec{q}+\vec{k}|}^{*}\left(\eta_{1}\right)\right|^{2}+n_{q}\left(1+n_{|\vec{q}+\vec{k}|}\right)\left|\int_{\eta_{R}}^{\eta} d \eta_{1} k \mathcal{D}_{C}\left(\eta, \eta_{1}, k\right) f_{q}^{*}\left(\eta_{1}\right) f_{|\vec{q}+\vec{k}|}\left(\eta_{1}\right)\right|^{2}+ \\
& \left.+n_{q} n_{|\vec{q}+\vec{k}|}\left|\int_{\eta_{R}}^{\eta} d \eta_{1} k \mathcal{D}_{C}\left(\eta, \eta_{1}, k\right) f_{q}^{*}\left(\eta_{1}\right) f_{|\vec{q}+\vec{k}|}^{*}\left(\eta_{1}\right)\right|^{2}\right]
\end{aligned}
$$

and

$$
S_{B}^{H}(\eta, k)=-i k^{2} F(\eta, \eta ; k)
$$

where $F\left(\eta, \eta^{\prime} ; k\right)$ satisfies the homogeneous differential equation

$$
\left[\frac{d^{2}}{d \eta^{2}}+k^{2}+\sigma(\eta) C(\eta) \frac{d}{d \eta}\right] F\left(\eta, \eta^{\prime}, k\right)+\int d \eta_{1}\left[\Pi^{l}\left(\eta_{1}\right) \delta\left(\eta-\eta_{1}\right)+\Pi_{R}\left(\eta, \eta_{1}\right)\right] F\left(\eta_{1}, \eta^{\prime}, k\right)=0
$$


with $\Pi^{l}(\eta), \Pi_{R}\left(\eta, \eta^{\prime}\right)$ being the one loop tadpole (local) and retarded (non-local) contributions transverse polarization [25].

We note that the function $F\left(\eta, \eta^{\prime} ; k\right)$ obeys the same equation as the transverse gauge mean field 25], but as it will be argued in detail below, its contribution to the spectrum of primordial magnetic fields generated during the phase transitions is subleading in the scalar coupling constant $\lambda$. The equation of motion (4.9) can be solved systematically in an expansion in powers of the non-equilibrium polarization,

$$
\begin{aligned}
& F(\eta, \eta ; k)=F^{(0)}(\eta, \eta ; k)+F^{(1)}(\eta, \eta ; k)+\mathcal{O}\left(\alpha^{2}\right), \\
& F^{(0)}\left(\eta, \eta^{\prime}, k\right)=\mathcal{D}_{H}\left(\eta, \eta^{\prime}, \vec{k}\right), \\
& F^{(1)}\left(\eta, \eta^{\prime}, k\right)=\int_{\eta_{R}}^{\eta} d \eta_{1} \mathcal{D}_{C}\left(\eta-\eta_{1}, \vec{k}\right) \int_{\eta_{R}}^{\eta} d \eta_{2}\left[\Pi^{l}\left(\eta_{2}\right) \delta\left(\eta_{1}-\eta_{2}\right)+\Pi_{R}\left(\eta_{1}, \eta_{2} ; \vec{k}\right)\right] \mathcal{D}_{H}\left(\eta_{2}-\eta^{\prime}, \vec{k}\right)+ \\
& +\quad\left(\eta \leftrightarrow \eta^{\prime}\right),
\end{aligned}
$$

where $\Pi^{l}, \Pi_{R}$ are the tadpole (local) and the retarded contribution from the one-loop transverse photon polarization respectively (for details see 25$]$ ).

These are given by 25]

$$
\Pi^{l}(\eta)=-i e^{2} N \int \frac{d^{3} q}{(2 \pi)^{3}} G_{>}(\eta, \eta, q) \quad, \quad \Pi_{R}\left(\eta_{1}, \eta_{2}, \vec{k}\right)=\left[\Pi^{>}\left(\eta_{1}, \eta_{2}, \vec{k}\right)-\Pi^{<}\left(\eta_{1}, \eta_{2}, \vec{k}\right)\right] \Theta\left(\eta_{1}-\eta_{2}\right)
$$

with

$$
\Pi_{>}\left(\eta_{1}, \eta_{2}, k\right)=2 i e^{2} N \int \frac{d^{3} q}{(2 \pi)^{3}} q^{2}\left(1-\cos ^{2} \theta\right) G_{>}\left(\eta_{1}, \eta_{2}, q\right) G_{>}\left(\eta_{1}, \eta_{2},|\vec{q}+\vec{k}|\right), \Pi_{<}\left(\eta_{1}, \eta_{2}, k\right)=\Pi_{>}\left(\eta_{2}, \eta_{1}, k\right) .
$$

The scalar propagator $G_{>}\left(\eta, \eta^{\prime} ; k\right)$ is constructed from the mode functions $f_{q}(\eta)$ that satisfy the mode equations (3.42) and is given by

$$
G_{>}\left(\eta_{1}, \eta_{2} ; k\right)=\frac{i}{2}\left[\left(1+n_{k}\right) f_{k}\left(\eta_{1}\right) f_{k}^{*}\left(\eta_{2}\right)+n_{k} f_{k}^{*}\left(\eta_{1}\right) f_{k}\left(\eta_{2}\right)\right]
$$

Therefore

$$
\Pi_{R}\left(\eta_{1}, \eta_{2}, \vec{k}\right)=4 e^{2} N \int \frac{d^{3} q}{(2 \pi)^{3}} q^{2}\left(1-\cos ^{2} \theta\right) \operatorname{Im}\left[G_{>}\left(\eta_{1}, \eta_{2}, q\right) G_{>}\left(\eta_{1}, \eta_{2},|\vec{q}+\vec{k}|\right)\right] \Theta\left(\eta_{1}-\eta_{2}\right) .
$$

This expression for the retarded self-energy must be contrasted with that of the contribution from $S_{B}^{I}(\eta, k)$ which requires the real part $\operatorname{Re}\left[G_{>}\left(\eta_{1}, \eta_{2}, q\right) G_{>}\left(\eta_{1}, \eta_{2},|\vec{q}+\vec{k}|\right)\right]$. This is an important difference, the long wavelength modes of largest amplitude in either phase given by (3.45) or by (3.59) are such that their phases are frozen, namely they do not depend on time, therefore the products $f_{q}\left(\eta_{1}\right) f_{|\vec{p}+\vec{q}|}\left(\eta_{2}\right)$ with only the growing mode solutions are real and such products will contribute only to $S_{B}^{I}(\eta, k)$. This freezing of phases is a consequence of the classicalization of the scalar field fluctuations [26].

We now argue that the contribution from $S_{B}^{H}$ is subleading. First of all, the term $F^{(0)}(\eta, \eta ; k)$ in eq. (4.10) is the solution of the homogeneous equation in absence of non-equilibrium fluctuations and leads to the local thermodynamic equilibrium contribution to the power spectrum, which is independent of the non-equilibrium generation mechanisms. This contribution has been analyzed in detail in ref. 25] and will be subtracted. In what follows we focus solely on the contribution from the non-equilibrium fluctuations.

For intermediate times after the phase transition during the spinodal stage $\eta_{c} \leq \eta<\eta_{n l}$, the long-wavelength mode functions are approximately given by eq.(3.45).

Near the end of the phase transition for $\eta \sim \eta^{\prime} \sim \eta_{n l}$ the leading order time dependence of the scalar Green's functions is approximately given by

$$
f_{k}(\eta) f_{k}\left(\eta^{\prime}\right) \propto \frac{1}{\lambda}
$$

where we used eq.(3.50). Thus, the contribution from the tadpole (local term in the self-energy) is of the order

$$
\Pi^{\operatorname{tad}}(\eta)_{\eta \sim \eta_{n l}} \propto \frac{e^{2}}{\lambda}+\text { subleading . }
$$


This estimate is consistent with the fact that the tadpole contribution is $e^{2}<\Phi^{\dagger} \Phi>$ and near the end of the phase transition the mean square root fluctuations of the scalar field probe the vacuum state, namely $\left\langle|\Phi|^{2}>\sim \mu^{2} / \lambda\right.$. Since the phases of these modes are frozen, there is no contribution from the leading order to the retarded polarization, since it requires the imaginary part of the product of propagators as displayed in eq. 4.13). Because of this cancellation of the leading term, the contribution from the retarded polarization bubble is of the same order as that of the tadpole [25, 48].

$$
\Pi_{R} \propto \frac{e^{2}}{\lambda} .
$$

A similar argument based on the sum rule (3.60) leads to the same conclusion in the scaling regime.

For late times, the $k \rightarrow 0$ limit of the retarded polarization exactly cancels the contribution from the tadpole [see eq.(6.12) in ref. [48]].

The contribution from $S_{B}^{I}$ is in both cases of $\mathcal{O}\left(1 / \lambda^{2}\right)$ since each long-wavelength mode function is of order $1 / \sqrt{\lambda}$ at the end of the spinodal stage or, by the sum rule (3.60) in the scaling regime. Thus we can safely neglect the contribution from $S_{B}^{H}$ to the magnetic spectrum.

Thus the leading contribution to the power spectrum generated by non-equilibrium fluctuations is given by

$$
S_{B}(\eta, k)=\left(1+2 n_{0}\right)^{2} \frac{\alpha N k^{2}}{\pi \sigma_{R}^{2}} e^{-\frac{2 k^{2}}{\sigma_{R}} \eta} \int_{0}^{\infty} q^{4} d q d(\cos \theta)\left(1-\cos ^{2} \theta\right)\left|\int_{\eta_{R}}^{\eta} e^{\frac{k^{2}}{\sigma_{R}} \eta_{1}} f_{q}\left(\eta_{1}\right) f_{|\vec{q}+\vec{k}|}\left(\eta_{1}\right) d \eta_{1}\right|^{2} .
$$

where $\theta$ is the angle between the vectors $\vec{q}$ and $\vec{k}$ and where we have replaced

$$
\left(1+2 n_{q}\right)\left(1+2 n_{|\vec{q}+\vec{k}|}\right) \simeq\left(1+2 n_{0}\right)^{2},
$$

since as highlighted in section (IIIB 1) the dynamics during both the spinodal stage as well as the scaling stage is dominated by the long-wavelength modes that acquire non-perturbatively large amplitudes.

The final form of the power spectrum generated by the non-equilibrium dynamics given by eq. (4.17) is the basis for the study of primordial magnetogenesis during the different stages after the phase transition.

\section{A. Magnetogenesis during the spinodal stage}

The long-wavelength mode functions in the spinodally unstable band are given by the expression (3.45).

The integral over $\eta_{1}$ for large $\eta$ can be computed integrating by parts in eq.(4.17) as an expansion in $1 /(\tilde{\mu} \eta)^{2}$. The integral is dominated by the upper limit, which leads to the cancellation of the exponentials that contain the conductivity.

The integrals over momenta and angles in eq.(4.17) can be done straightforwardly when the mode functions are given by eq.(3.45). Thus from eq.(4.17) whe obtain the following expression for the spectrum of magnetic fields generated by the non-equilibrium fluctuations

$$
S_{B}\left(k, \eta \sim \eta_{n l}\right)=\frac{512 \pi^{\frac{9}{2}} N \alpha k^{2}}{\lambda^{2} \sigma_{R}^{2} \tilde{\mu}^{4} \xi^{5}\left(\eta_{n l}\right)} e^{-\frac{1}{4} k^{2} \xi^{2}\left(\eta_{n l}\right)}\left[1+\mathcal{O}\left(\frac{1}{\ln \frac{1}{\lambda}}\right)\right] .
$$

where $\xi(\eta)$ is given by eq. (3.53). In obtaining this result we used the following

$$
\frac{\left[1+2 n_{0}\right]^{2}\left|a_{0}\right|^{4}}{\left(\tilde{\mu} \eta_{n l}\right)^{6}} e^{2\left(\tilde{\mu} \eta_{n l}\right)^{2}}=\frac{1024 \pi^{5}}{\lambda^{2} \tilde{\mu}^{2}}
$$

[see eq. [3.48] ] and the identities [46],

$$
\int_{-1}^{+1} d x\left(1-x^{2}\right) e^{-\frac{4 q k x}{\tilde{\mu}^{2}} \ln \tilde{\mu} \eta}=\frac{\tilde{\mu}^{6}}{16(q k \ln \tilde{\mu} \eta)^{3}}\left\{\frac{4 q k}{\tilde{\mu}^{2}} \ln (\tilde{\mu} \eta) \cosh \left[\frac{4 q k}{\tilde{\mu}^{2}} \ln \tilde{\mu} \eta\right]-\sinh \left[\frac{4 q k}{\tilde{\mu}^{2}} \ln \tilde{\mu} \eta\right]\right\}
$$

and

$$
\int_{0}^{\infty} q d q e^{-\xi^{2} q^{2}}\left[\xi^{2} q k \cosh \left(\xi^{2} q k\right)-\sinh \left(\xi^{2} q k\right)\right]=\frac{\sqrt{\pi}}{8} k^{3} \xi e^{\frac{1}{4} k^{2} \xi^{2}}
$$


Notice that the magnetic field spectrum (4.18) is independent on the amplitude $\left|a_{0}\right|$ and on the initial occupation $\left(1+2 n_{0}\right)^{2}$. Therefore this result is quite robust.

This result is the same as for the Minkowski space-time (see eq.(7.47) in ref. 25]), except for a multiplicative factor $\tilde{\mu}^{6} \xi^{6}\left(\eta_{n l}\right) \simeq 8 \ln ^{3}\left(\ln \frac{1}{\lambda}\right)$ and the expression for the correlation length in the radiation dominated universe (3.53).

As in Minkowki space-time, the presence of a high conductivity plasma severely hinders the generation of magnetic fields. However, a noteworthy aspect is that up to the non-linear time the magnetic field is still correlated over the size of the scalar field domains rather than the diffusion length $\xi_{\text {diff }} \approx \sqrt{\eta / \sigma_{R}}$. The diffusion scale determines the spatial size of the region in which magnetic fields are correlated in the absence of non-equilibrium generation. The ratio between the domain size $\xi(\eta)$ given by eq.(3.53) and the diffusion length scale $\xi_{\text {diff }}(\eta)$ is given by

$$
\frac{\xi\left(\eta_{n l}\right)}{\xi_{d i f f}\left(\eta_{n l}\right)} \simeq \frac{2}{\tilde{\mu}} \sqrt{\frac{\sigma_{R} \ln \left(\tilde{\mu} \eta_{n l}\right)}{\eta_{n l}}} \sim\left(\frac{M_{P l}^{2} \ln \frac{1}{\lambda}}{\mu^{2}}\right)^{\frac{1}{4}} \gg 1 .
$$

Where we have used the relations (3.3), (3.4), (3.20) and (3.48). Thus an important conclusion of this study is that the magnetic fields generated via spinodal decomposition are correlated over regions comparable to the size of scalar field domains which are much larger than the diffusion scale.

The spectrum for the electric field can be obtained from that of the magnetic field by simply replacing $k \mathcal{D}_{C} \rightarrow \dot{\mathcal{D}}_{C}$. In the soft regime and for time scales $\frac{1}{\sigma_{R}} \ll \eta \ll \frac{\sigma_{R}}{k^{2}}$ we have $\dot{\mathcal{D}}_{C} \simeq-k^{2} / \sigma_{R}^{2}$ whereas $k \mathcal{D}_{c} \simeq k / \sigma_{R}$. Therefore the electric field spectrum is suppressed by a factor $k^{2} / \sigma_{R}^{2}$ with respect to the magnetic field, namely

$$
S_{E}^{\sigma_{R}}(t, k)=\frac{k^{2}}{\sigma_{R}^{2}} S_{B}^{\sigma_{R}}(t, k) .
$$

Thus, in a high temperature plasma with large conductivity the non-equilibrium processes favor the generation of magnetic photons instead of electric photons, and again equipartition is not fulfilled.

The energy density on large scales $\geq L$ again can be computed in closed form in the limits $L \gg \xi\left(t_{n l}\right)$ and $L \ll \xi\left(t_{n l}\right)$. We find in the first case from eq.4.3),

$$
\Delta \rho_{B}\left(\eta_{n l}, L\right)=\frac{2^{13} \pi^{\frac{15}{2}}}{5 \lambda^{2}} \frac{N \alpha}{\left[\tilde{\mu} \xi\left(\eta_{n l}\right)\right]^{4} \sigma_{R}^{2} \xi\left(\eta_{n l}\right) L} \frac{1}{L^{4}} \quad ; \quad L \gg \xi\left(t_{n l}\right)
$$

We find for the opposite case,

$$
\Delta \rho_{B}\left(\eta_{n l}, L\right)=\frac{3 \times 2^{10} \pi^{3} N \alpha}{\lambda^{2} \tilde{\mu}^{4} \sigma_{R}^{2} \xi^{10}\left(\eta_{n l}\right)} ; L \ll \xi\left(t_{n l}\right) .
$$

The ratio of the magnetic energy density on scales larger than $L$ at the spinodal time and the total radiation energy given by the Stefan-Boltzman law $\rho_{\gamma}=\pi^{2} T_{n l}^{4} / 15$ results,

$$
r\left(\eta_{n l}, L\right)=\frac{\Delta \rho_{B}\left(\eta_{n l}, L\right)}{\rho_{\gamma}}=\frac{3 \times 2^{13} \pi^{\frac{11}{2}}}{\lambda^{2}} \frac{N \alpha}{\left[\tilde{\mu} \xi\left(\eta_{n l}\right)\right]^{4} \sigma_{R}^{2} \xi\left(\eta_{n l}\right) L} \frac{1}{\left(L T_{R}\right)^{4}} \quad ; \quad L \gg \xi\left(t_{n l}\right) .
$$

This result is the same as for the Minkowski space-time (see eq.(7.53) in ref.[25]), except for a multiplicative factor $\tilde{\mu}^{6} \xi^{6}\left(\eta_{n l}\right) \simeq 8 \ln ^{3}\left(\ln \frac{1}{\lambda}\right)$ and the expression for the correlation length in the radiation dominated universe (3.53).

The factor $\left(L T_{R}\right)^{-4}$ is purely dimensional and is ultimately the determinining factor for the strength of the generated magnetic fields on a given scale. These combinations are invariant under cosmological expansion and are determined by the ratio of the scales of interest today (galactic) to the thermal wavelength (today) of the cosmic microwave background radiation at the Wien peak. In particular $L T_{R} \sim 10^{25}$ for $L \sim 1 \mathrm{Mpc}$ (today) [see eq.(3.16)].

It is clear that the production during this regime is extremely small, due to the large values of $(L T)^{4}$ and of the ratio $\sigma_{R}^{2} / \mu^{2}$. In order to obtain an estimate for the amplitude of the seed magnetic field, we consider the following set of parameters: $\lambda=10^{-2}, \alpha=10^{-2}, \mu=10^{14} \mathrm{GeV}, T_{R}=10^{16} \mathrm{GeV}$ (corresponding to a critical temperature $\left.T_{c}=10^{15} \mathrm{GeV}\right)$. We then obtain,

$$
r(L=1 M p c) \sim 10^{-157} .
$$

Therefore, the amplitude of the magnetic field generated during the spinodal stage is completely negligible. This result is similar to the result obtained in Minkowski space-time in ref. [25] and is expected on the basis of dimensional analysis. 


\section{B. Magnetogenesis from the scaling regime}

In the scaling regime $\eta>>\eta_{n l}$ the spectrum of the magnetic field is given by eq. (4.17) with the mode functions in the scaling regime given by eq. (3.59).

The final expression for the leading contribution, given by eq. (4.17) reveals a noteworthy aspect. As we have argued above, the modes $k$ of astrophysical relevance today, were well outside the horizon during the radiation dominated era between reheating and the QCD phase transition. The mode functions eq. (4.17) attain the largest amplitude at long times for $x=q \eta \leq 2-3$, thus momenta in the polarization loop that are within the horizon lead to generation of magnetic fields with long-wavelengths well outside the horizon. This we believe, is an important mechanism, loop corrections lead to a coupling between modes inside the horizon with those outside. Thus in this manner, causal fluctuations can actually lead to the generation of fields with wavelengths much larger than the horizon.

Since $k \eta \ll 1$ the power spectrum eq.(4.17) takes the following form using the scaling mode functions eq.(3.59),

$$
S_{B}(\eta, k)=\left(1+2 n_{0}\right)^{2} \frac{\alpha N k^{2}}{\pi \sigma_{R}^{2}}\left|A_{0}\right|^{2} \int_{0}^{\infty} d q \int_{\eta_{R}}^{\eta} J_{2}\left(q \eta_{1}\right) \eta_{1} d \eta_{1} \int_{\eta_{R}}^{\eta} J_{2}\left(q \eta_{2}\right) \eta_{2} d \eta_{2} I\left(q, k, \eta_{1}, \eta_{2}\right),
$$

where we set the exponentials equal to unity in eq.(4.17) since $k \eta \ll 1$ and $k \ll \sigma_{R}$ and

$$
I\left(q, k, \eta_{1}, \eta_{2}\right) \equiv \int_{-1}^{+1} d x \frac{1-x^{2}}{\left(q^{2}+k^{2}-2 k q x\right)^{2}} J_{2}\left(\sqrt{q^{2}+k^{2}-2 k q x} \eta_{1}\right) J_{2}\left(\sqrt{q^{2}+k^{2}-2 k q x} \eta_{2}\right) .
$$

Using the summation theorem [46]

$$
J_{2}\left(\sqrt{q^{2}+k^{2}-2 k q x} \eta\right)=\frac{4\left(q^{2}+k^{2}-2 k q x\right)}{q^{2} k^{2} \eta^{2}} \sum_{l=0}^{\infty}(l+2) J_{l+2}(q \eta) J_{l+2}(k \eta) C_{l}^{2}(x)
$$

where the $C_{l}^{2}(x)$ are Gegenbauer polynomials. For $k \eta \ll 1$ the $l=0$ terms dominate and we can use the small argument behaviour of the Bessel functions $J_{2}(k \eta)=\frac{1}{8}(k \eta)^{2}\left[1+\mathcal{O}\left(k^{2} \eta^{2}\right)\right]$. We finally obtain,

$$
I\left(q, k, \eta_{1}, \eta_{2}\right)=\frac{4}{3 q^{4}} J_{2}\left(q \eta_{1}\right) J_{2}\left(q \eta_{2}\right)\left[1+\mathcal{O}\left(k^{2} \eta^{2}\right)\right] .
$$

Inserting eq.(4.29) into eq.4.28) yields

$$
S_{B}(\eta, k)=\left(1+2 n_{0}\right)^{2} \frac{\alpha N k^{2}}{3 \pi \sigma_{R}^{2}}\left|A_{0}\right|^{2} \int_{0}^{\infty} \frac{d q}{q^{4}}\left\{\eta^{2}\left[J_{2}^{2}(q \eta)-J_{1}(q \eta) J_{3}(q \eta)\right]-\left(\eta \rightarrow \eta_{R}\right)\right\}^{2}\left[1+\mathcal{O}\left(k^{2} \eta^{2}\right)\right]
$$

where we used the formula 46$]$

$$
\int_{0}^{y} z J_{2}^{2}(\beta z) d z=\frac{y^{2}}{2}\left[J_{2}^{2}(\beta y)-J_{1}(\beta y) J_{3}(\beta y)\right]
$$

Since $\eta \gg \eta_{R}$ we can neglect the terms with $\eta_{R}$ and we find, for $k \ll \eta^{-1}$

$$
S_{B}(\eta, k)=\mathcal{D} \frac{\alpha N}{\lambda^{2}} \frac{k^{2}}{\sigma_{R}^{2}} \mu^{4} H_{R}^{4} \eta^{7}\left[1+\mathcal{O}\left(k^{2} \eta^{2}\right)\right] \quad ; \quad \mathcal{D}=48.61 \ldots
$$

where we used eq.(3.61) and we computed numerically the integral

$$
\int_{0}^{\infty} \frac{d x}{x^{4}}\left[J_{2}^{2}(x)-J_{1}(x) J_{3}(x)\right]^{2}=0.0005295 \ldots
$$

This integral is dominated by the region $x \geq 1$, namely, by modes that are inside the horizon. From the estimate (3.24), the corrections $\mathcal{O}\left(k^{2} \xi_{\text {diff }}^{2}(\eta)\right)$ are truly negligible between reheating and the QCD phase transition.

The dependence on the conformal time $\sim \eta^{7}$ is a direct consequence of the scaling form of the solution for the mode functions. The strong time dependence is a consequence of the causal relaxation of the Goldstone fields, a result of the phase ordering kinetics that entails that the size of the domains grow with the horizon.

The spectrum eq.(4.32) exhibits the following important features: 
- i: The exponential associated with the diffusion length cancels out, a reflection that the long time behavior of the integrals above are dominated by the upper limit. Hence the final result for the spectrum does not feature the exponential suppression with the diffusion length.

- ii: The result for the spectrum only depends on the initial amplitud $A_{0}$ and initial occupation number $n_{0}$ in the combination $\left|A_{0}\right|^{2}\left(1+2 n_{0}\right)$ which is constrained by the sum rule eq.(3.60). Hence the final spectrum is insensitive to the initial conditions on the mode functions or occupations, which in principle carry information of the early history beginning from the inflationary stage. This is a consequence of the scaling solution being a fixed point of the dynamics of the scalar field [26, 27, 28].

- iii: A noteworthy result is that superhorizon magnetic fields are generated by the non-equilibrium dynamics of modes inside but near the Hubble radius. This is a consequence of the polarization loop, wherein the propagators correspond to momenta $q$ and $|\vec{q}+\vec{k}|$. The momenta $k$ corresponding the wavevector (scale) of the magnetic field is such that the wavelength is larger than the Hubble radius, but the momenta $q$ corresponding to the charged scalar field fluctuations are inside the horizon. The correlation length of the charged scalar field is of the order of the Hubble radius. Thus acausal, superhorizon magnetic fields are generated by loop effects.

In order to reveal the enhancement during the scaling regime in a more transparent manner, it is convenient to use the relations (3.3), (3.13), (3.40) and the explicit expression for the conductivity (3.63) in the form

$$
\sigma_{R}=c[\alpha, N] \frac{N T_{R}}{\alpha} \quad, \quad c[\alpha, N] \equiv \frac{\mathcal{C}}{\ln \left[\frac{1}{\alpha N}\right]} \sim \mathcal{O}(1) .
$$

Then, the ratio $r(L, \eta)$ for $L \gg \eta$ is given by

$$
r(\eta, L)=\frac{240 \pi \mathcal{D} \alpha^{3}}{N c^{2}[\alpha, N]\left[L T_{R}\right]^{5}}\left(\frac{\mu}{\sqrt{\lambda} T(\eta)}\right)^{4}\left(\frac{M_{*}}{T(\eta)}\right)^{3} .
$$

where $\mathcal{D}$ is given in eq. (4.32). We note that in the final result (4.34) there is no dependence on the reheating temperature but only on the scale of symmetry breaking $\mu$, the temperature at the time $\eta$ and the scalar and gauge couplings. This is expected since the non-equilibrium processes begin in earnest after the phase transition, local thermal equilibrium prevailed between the time of reheating and the phase transition.

The dependence on the scalar self coupling $\propto 1 / \lambda^{2}$ is a hallmark of the non-perturbative nature of the growth of unstable modes and spinodal decomposition, it is ubiquitous in the non-equilibrium dynamics of phase transitions 26 , 40, 41].

Large scale magnetogenesis is more efficient for large symmetry breaking scale $\mu$, since the larger the symmetry breaking scale, the longer lasts the scaling stage.

Consider for instance the case in which the symmetry breaking scale $\mu \sim 10^{13} \mathrm{Gev}$ and $\lambda \sim \alpha \sim 10^{-2}$, corresponding to a critical temperature of order of a GUT scale $T_{c} \sim 10^{15} \mathrm{GeV}$ and suppose that the scaling regime lasts until the electroweak phase transition scale, i.e. $\eta$ is such that $T(\eta)=T_{E W} \sim 10^{2} \mathrm{GeV}$. Then the factor

$$
\left(\frac{\mu}{\sqrt{\lambda} T_{E W}}\right)^{4}\left(\frac{M_{*}}{T_{E W}}\right)^{3} \sim 10^{100}
$$

compensates for the factor $\left(L T_{R}\right)^{-5}$. Taking $N$ and $g_{*}$ of the order of 10 (these values are taken as representative and they can be changed simply in the final expressions) we can write the expression for the ratio as

$$
r(T(\eta), L) \simeq 10^{-34}\left(\frac{L}{1 \mathrm{Mpc}}\right)^{-5}\left(\frac{T_{E W}}{T(\eta)}\right)^{7}
$$

Therefore

$$
r(T(\eta), L) \sim\left\{\begin{array}{l}
10^{-34} \text { at the EW transition } \\
10^{-14} \text { at the QCD transition }
\end{array}\right.
$$

Thus if the scaling regime lasts until a time between the EW and the QCD phase transitions the amplitude of the large scale magnetic fields is within the range necessary to be amplified by some dynamo models. The amplitude of the seed magnetic field is strongly dependent on the duration of the scaling regime. We have only focused on a scaling regime terminating either at the EW or QCD phase transition since there will surely be new phenomena associated with these that must be included in the dynamics of magnetogenesis. 


\section{Discussion}

- Validity of the approximations: There are two main approximations that were used to obtain the results quoted above, i) the long-wavelength approximation $k \eta \ll 1$ and ii) the weak coupling approximation. We now provide an estimate of the reliability of both these approximations to establish the limit of validity of our results.

i): Long-wavelength approximation: In order to reach our final result for the rate $r(L, \eta)$ we have explicitly used a series of approximations which are valid for long wavelengths but whose validity must be checked before we reach any conclusion regarding the spectrum at small scales. In particular we must address the limits of applicability of the result eq.(4.34). This result has been obtained by integrating the magnetic spectrum on scales $0<k<k_{\max }$ with $k_{\max }=2 \pi / L_{\min }$; the formula for the magnetic spectrum was valid in the limit

$$
k_{\max } \eta_{\max } \ll 1 .
$$

In order to provide an estimate may take for $\eta_{\max }$ to be the (conformal) time at which the EW phase transition occurs, namely $\eta_{E W} \sim 1 \mathrm{GeV}^{-1}$. As discussed in the introduction, we are considering a situation in which the magnetic field is considered as a perturbation of a pre-existing thermal blackbody background. For consistency this requires that,

$$
r\left(L_{\min }, \eta_{W E}\right) \ll 1
$$

This relation translates in a condition

$$
k_{\max } \eta_{\max } \ll\left[\frac{C N \alpha^{3}}{24^{2} c^{2}}\left(\frac{T_{c}}{T_{E W}}\right)^{4}\left(\frac{T_{E W}}{M_{*}}\right)^{2}\right]^{-1 / 5} .
$$

For $T_{c} \sim 10^{15} \mathrm{GeV}$ this gives $k_{\max } \eta_{\max } \ll 0.0176$ which in turns translate into

$$
L \gg L_{\min } \sim 70 \mathrm{fm} .
$$

However, this is the comoving length normalized at the reheating time. In order to convert to the present time, we have to take in account the redshift

$$
z_{R}=\frac{T_{R}}{T_{0}} \simeq 4 \times 10^{28} \text { for } T_{R} \sim 10^{15} \mathrm{Gev} ;
$$

this gives

$$
\left.L_{\text {min }}\right|_{\text {today }} \sim 0.1 \mathrm{pc} .
$$

Thus, the approximations invoked are reliable to estimate the amplitude of primordial seeds on galactic scales or larger, today.

ii): Weak electromagnetic coupling: In order to study the amplitude for much smaller scales the calculations must be done without the long wavelength approximations invoked above. In this case we must expect a breakdown of perturbation theory and we cannot give a reliable estimate in the present framework. Furthermore, for scales well inside the Hubble radius, microphysical processes not included in our approximations, such as scattering between charged fields and between charged and gauge fields must be included. These processes will restore equilibrium between the different fields, if there is a substantial transfer of power from the charged fluctuations to the radiation field, this may lead to a change in the equation of state and the full backreaction on the metric must be included. At longer time scales the effects of the backreaction of the gauge fields on the dynamics of the scalar field, as well as the non-equilibrium contributions to equation of state and the Friedmann equations must be included self-consistently.

- Generation on short distance scales: For scales well inside the horizon during the scaling regime, namely $q \eta \gg 1$, we must account for causal microscopic processes that tend to equilibrate the electromagnetic fields generated by the non-equilibrium processes. In order to understand these processes we must look at the kinetics of equilibration. The mode functions for wavevectors well inside the horizon are Minkowski-like, of the form

$$
f_{q}(\eta)=\frac{\alpha_{q}}{\sqrt{q}} e^{-i q \eta}+\frac{\beta_{q}}{\sqrt{q}} e^{i q \eta},
$$


where the coefficients $\alpha_{q}, \beta_{q}$ must be determined from a full numerical evolution. However the constancy of the Wronskian entails that

$$
\left|\alpha_{q}\right|^{2}-\left|\beta_{q}\right|^{2}=1
$$

which suggests the identification $\left|\alpha_{q}\right| \equiv 1+\mathcal{N}_{q} ;\left|\beta_{q}\right| \equiv \mathcal{N}_{q}, \mathcal{N}_{q}$ is the number of (asymptotic) quanta created during the time evolution. This form of the asymptotic mode functions leads to the equipartition between the electric and magnetic field generation, since spatial and time derivatives are the same. In turn this entails that we can understand the generation of electric and magnetic fields by obtaining a kinetic equation for the number of photons. Such kinetic equation must necessarily be of the form

$$
\frac{d N_{k}(\eta)}{d \eta}=\left[1+N_{k}(\eta)\right] \Gamma_{k}^{>}(\eta)-N_{k}(\eta) \Gamma_{k}^{<}(\eta)
$$

which displays the familiar gain minus loss contributions in terms of the forward and inverse rates. Eventually a steady state will be reached which will describe a stationary distribution of photons. The computation of the forward $\left[\Gamma_{k}^{>}(\eta)\right]$ and inverse $\left[\Gamma_{k}^{<}(\eta)\right]$ require a detailed knowledge of the distribution $\mathcal{N}_{q}[48]$ since these generalized rates are functionals of these occupation numbers. Clearly such computation lies beyond the scope of this article and is a task that we will undertake elsewhere. However, the kinetic equations above will tend to an equilibrated state of local thermodynamic equilibrium.

- Effect on the LSS: It is important to estimate the effect of the magnetic field on scales corresponding to those of the last scattering surface, which today are $L_{L S S} \sim 100 \mathrm{Mpc}$. From eq. (4.36) we see that at the electroweak temperature $r\left(T_{E W}, L_{L S S}\right) \sim 10^{-44}$, taking the fourth root we can provide an estimate of the temperature fluctuation induced by the primordial magnetic field $\delta T /\left.T\right|_{L S S} \sim\left[r\left(T_{E W}, L_{L S S}\right)\right]^{\frac{1}{4}} \sim 10^{-11}$ which is negligible compared to the $\mathrm{CMB}$ temperature fluctuation at this scale $\sim 10^{-5}$. On the other hand, a similar estimate at the time of the QCD phase transition gives $\delta T /\left.T\right|_{L S S} \sim 10^{-6}$ which is marginally compatible with the current observations. Thus the reliability of the approximation of weak gauge coupling combined with the effects on the temperature anisotropy at the last scattering surface seem to lead us to conclude that if a phase transition during a radiation dominated era occurs near the GUT scale and results in a scaling stage, our results for primordial magnetogenesis will be reliable down to the scale of electroweak symmetry breaking.

\section{CONCLUSIONS}

In this article we studied large scale primordial magnetogenesis during a phase transition in the radiation dominated era after reheating in a model of $N$-charged scalars coupled to an abelian gauge field. The spectrum of the magnetic field generated during the non-equilibrium evolution was computed using the formulation recently introduced in ref. 25]. The dissipative effects of the conductivity are included by separating the contribution from hard modes (with momenta of order $T$ ) to the polarization tensor of the gauge fields. These modes are always in local thermodynamic equilibrium. The non-perturbative, non-equilibrium dynamics of the scalar field after the phase transition was studied in the large $N$ limit. The dynamics after the phase transition features two distinct stages: an early and intermediate time, spinodal stage, which is dominated by the growth of long-wavelength fluctuations, followed by a scaling regime during which the scalar field becomes correlated over horizon-sized domains. During both regimes, strong nonequilibrium fluctuations lead to large current-current correlation functions which entail the generation of magnetic fields. The scaling regime is the most effective for primordial magnetogenesis since this stage lasts the longest. During this stage magnetic fields with superhorizon wavelengths are generated via loop effects, the scalar field momenta in the polarization loop corresponds to wavelengths of the order of or shorter than the horizon. Thus causal scalar field fluctuations lead to the generation of magnetic fields on superhorizon scales. The generation of magnetic field is hindered by the large conductivity of the plasma and equipartition between electric and magnetic fields does not hold. The spectrum of the primordial magnetic field is insensitive to the magnetic diffusion length which is sub-horizon during the radiation era.

Our final result for the spectrum generated during the scaling regime is given by eq.44.32). The ratio of the energy density of the magnetic fields on scales larger than $L$ to the energy density in the cosmic background radiation $r(L, \eta)=\rho_{B}(L, \eta) / \rho_{c m b}(L, \eta)$ is given by equation (4.34). For values of $N$, and the gauge coupling consistent with particle physics models we find that

$$
r(T(\eta), L) \simeq 10^{-34}\left(\frac{L}{1 \mathrm{Mpc}}\right)^{-5}\left(\frac{T_{E W}}{T(\eta)}\right)^{7} .
$$


Therefore,

$$
r(T(\eta), L) \sim\left\{\begin{array}{l}
10^{-34} \text { at the EW transition } \\
10^{-14} \text { at the QCD transition }
\end{array}\right.
$$

Therefore, the large scale primordial magnetic fields generated during the scaling stage after a phase transition may be a plausible mechanism to generate primordial magnetic fields which will be further amplified by the collapse of protogalaxies and by astrophysical dynamos.

Probably a phase transition at a temperature much larger than the electroweak leading to a scaling regime lasting until the QCD phase transition is ruled out by the temperature inhomogeneities at the last scattering surface. Furthermore the generation of electromagnetic fields on sub-horizon scales requires a full kinetic equation that incorporates the microscopic causal processes that lead to thermalization, the study of these is beyond the scope of this article.

Magnetogenesis after the QCD phase transition? The model that we studied here is assumed to describe the robust features from the non-equilibrium dynamics of a charged sector coupled to a (hyper) charge gauge field. GUT's or SUSY theories may provide the corresponding framework.

However we now argue that precisely the model studied here can actually describe the non-equilibrium dynamics after the QCD phase transition(s). After hadronization and chiral symmetry breaking most of the hadrons produced will be pions, at least this is the experimental situation in ultrarelativistic heavy ion collisions. Neglecting the charge form factor (which is justified for momenta much smaller than the $\rho$ meson mass $m_{\rho} \sim 770$ Mev) the charged pions couple to the electromagnetic field with minimal coupling. The chiral transition is conjectured to be in the same universality class as the $O(4)$ linear sigma model 30]. Thus the model presented in this article, is the low energy effective field theory for the triplet of pions, two charged and one neutral. Thus we conjecture that the study in this article does describe the generation of magnetic fields by long-wavelength pions. Therefore the analysis of this article can apply to magnetogenesis during the chiral phase transition in QCD. While the charged pions couple to electromagnetism via the minimal coupling in the long-wavelength limit, the neutral pion couples to the electromagnetic field through the chiral anomaly $\pi^{0} \rightarrow 2 \gamma$ and such process will also produce magnetic fields. We will study the possibility of large scale primordial magnetogenesis during the chiral phase transition in QCD in a forthcoming article.

Acknowledgements: The authors thank M. Giovannini, for useful discussions. D. B. and M. S. thank N.S.F. for support through grants PHY-9988720 and NSF-INT-9815064.

[1] E. N. Parker, Cosmical Magnetic fields, Clarendon, Oxford, 1979. Ya. B. Zel'dovich, A.A. Ruzmikin, D.D. Sokoloff, Magnetic Fields in Astrophysics, Gordon and Breach, New York, 1983.

[2] P. P. Kronberg, Rep. Prog. Phys. 57, 325 (1994).

[3] D. Grasso and H. R. Rubinstein, Phys. Rep. 348, 163 (2001).

[4] L. Widrow, Revs. of Mod. Phys. 74, 775 (2002).

[5] M. Giovannini, hep-ph/0208152 to appear in the Proceedings of the 7th. Colloque Cosmologie, H J de Vega and N. Sanchez, editors. M. Giovannini, hep-ph/0111220

[6] For a recent review on observations see: J.-L. Han and R. Wielebinski, astro-ph/0209090

[7] D. Grasso, hep-ph/0002197. P. Elmfors, K. Enqvist and K. Kainulainen, Phys. Lett. B440, 269 (1998); M. Giovannini and M. E. Shaposhnikov, Phys. Rev. D57, 2186 (1988).

[8] T. Kahniashvili, A. Kosowsky, A. Mack and R. Durrer, astro-ph/0011095 (2000); A. Mack, T. Kahniashvili, A. Kosowsky, Phys. Rev. D65 123004 (2002); R. Durrer, P.G. Ferreira, T. Kahniashvili, Phys. Rev. D61, 043001 (2000); J. Adams, U. H. Danielsson, D. Grasso, H. Rubinstein, Phys. Lett. B388, 253 (1996).

[9] A. D. Dolgov, hep-ph/0110293 (2001).

[10] C. J. Hogan, Phys. Rev. Lett. 51, 1488 (1983).

[11] M. S. Turner and L. M. Widrow, Phys. Rev. D37, 2743 (1988).

[12] W. D. Garretson, G. B. Field, S. M. Carroll, Phys. Rev. D46, 5346 (1992); B. Ratra, Ap. J. 391, L1, (1992).

[13] M. Giovannini and M. E. Shaposhnikov, Phys. Rev. D 62, 103512 (2000) hep-ph/0004269.

[14] M. Giovannini and M. E. Shaposhnikov, hep-ph/0011105 (2000)

[15] A. C. Davis, K. Dimopoulos, T. Prokopec and O. Tornkvist, Phys. Lett. B 501, 165 (2001); K. Dimopoulos, T. Prokopec, O. Tornkvist and A. C. Davis, Phys. Rev. D 65, 063505 (2002); T. Prokopec, astro-ph/0106247

[16] E. A. Calzetta and A. Kandus, Phys. Rev. D 65, 063004 (2002) astro-ph/0110341. E. A. Calzetta, A. Kandus and F. D. Mazzitelli, Phys. Rev. D 57, 7139 (1998) astro-ph/9707220.

[17] B. A. Bassett, G. Pollifrone, S. Tsujikawa, F. Viniegra; Phys. Rev. D63 103515 (2001).

[18] F. Finelli and A. Gruppuso, Phys. Lett.B502 216 (2001); F. Finelli, astro-ph/0007290

[19] D. S. Lee, W. l. Lee and K. W. Ng, Phys. Lett. B542, 1 (2002).

[20] A. Dolgov, Phys. Rev. D 48, 2499 (1993).

[21] A.-C.Davis and K. Dimopoulos, Phys. Rev. D55 7398, (1997). T. Vachaspati, Phys. Lett. B 265, 258 (1991). 
[22] K. Enqvist and P. Olesen, Phys. Lett. B 319, 178 (1993); ibid 329, 195 (1994); K. Enqvist, in Strong and Electroweak Matter '97, Ed. F. Csikor, Z. Fodor, World Scientific, Singapore, 1998.

[23] G. Baym, D. Bodeker, L. McLerran, Phys. Rev. D53 662 (1996).

[24] J. Ahonen and K. Enqvist, Phys. Rev. D57, 664 (1998).

[25] D. Boyanovsky, H.J. de Vega and M. Simionato, Magnetic field generation from non-equilibrium phase transitions, Phys. Rev. D 67, 023502 (2003).

[26] D. Boyanovsky and H. J. de Vega, Phys. Rev. D 61, 105014 (2000); D. Boyanovsky, H. J. de Vega and R. Holman, Non-equilibrium phase transitions in condensed matter and cosmology: spinodal decomposition, condensates and defects, in: Topological Defects and the Non-Equilibrium Dynamics of Symmetry Breaking Phase Transitions, Eds. Y. M. Bunkov and H. Godfrin, Nato Science Series, Kluwer, 2000.

[27] N. Turok and D. N. Spergel, Phys. Rev. Lett. 66, 3093 (1991); D. N. Spergel, N. Turok, W. H. Press and B. S. Ryden, Phys. Rev. D43, 1038 (1991).

[28] R. Durrer, M. Kunz, A. Melchiorri, Phys. Rep. 3641 (2002); R. Durrer, M. Kunz, A. Melchiorri, Phys. Rev. D59 123005 (1999).

[29] J. F. Donohue, E. Golovich, B. R. Holstein, 'Dynamics of the Standard Model', Cambridge Univ. Press, 1992, pages 158, 159, 170, 171.

[30] K. Rajagopal and F. Wilczek, Nucl. Phys. B399, 395 (1993); Nucl. Phys. B404, 577 (1993); K. Rajagopal, in 'Quark-Gluon Plasma 2', Ed. by R. C. Hwa, World Scientific, Singapore, (1995).

[31] D. Boyanovsky, H. J. de Vega, R. Holman et S. Prem Kumar, Phys. Rev. D56, 5233 (1997) and D56, 3929 (1997).

[32] D. Boyanovsky, D. Brahm, R. Holman and D. S. Lee, Phys. Rev. D54, 1763 (1996); D. Boyanovsky, H. de Vega and S.-Y. Wang, Phys. Rev. D61, 065006 (2000).

[33] A. Achúcarro and T. Vachaspati, Phys. Rep. 327, 347 (2000).

[34] A. Achúcarro, J. Borrill and A. R. Liddle, Phys. Rev. D57, 3742 (1998); Phys. Rev. Lett. 82, 3742 (1999); Physica B255, 116 (1998).

[35] M. Hindmarsh, Nucl.Phys. B392, 461 (1993).

[36] D. Boyanovsky, D.-S. Lee and A. Singh, Phys. Rev. D48, 800, (1993); see also ref. 26].

[37] D. Boyanovsky, H. J. de Vega, R. Holman and M. Simionato, Phys. Rev. D60 (1999) 065003; D. Boyanovsky and H. J. de Vega, Phys. Rev. D59 (1999) 105019; S.-Y. Wang, D. Boyanovsky, H.J. de Vega, and D.-S. Lee, Phys. Rev. D 62, 105026 (2000).

[38] G. Baym and H. Heiselberg, Phys. Rev. D56, 5254 (1997).

[39] P. Arnold, G. D. Moore, L. G. Yaffe, JHEP 0011, 1 (2000).

[40] D. Boyanovsky, H.J. de Vega and R. Holman, Phys. Rev. D49, 2769 (1994); D. Boyanovsky, D. Cormier, H. J. de Vega, R. Holman, A. Singh, M. Srednicki, Phys. Rev. D56,1939 (1997).

[41] D. Boyanovsky, D.-S. Lee and A. Singh, Phys. Rev. D48, 800, (1993); D. Boyanovsky, H. J. de Vega, R. Holman, D.S-Lee and A. Singh, Phys. Rev. D51, 4419 (1995), D. Boyanovsky, D. Cormier, H.J. de Vega and R. Holman, Phys. Rev D55, 3373 (1997); D. Boyanovsky, D. Cormier, H. J. de Vega, R. Holman and S. Prem Kumar, Phys. Rev. D57, 2166 (1998); D. Boyanovsky, H. J. de Vega and R. Holman, Proceedings of the Second Paris Cosmology Colloquium, Observatoire de Paris, June 1994, pp. 127-215, H. J. de Vega and N. Sánchez, Editors, World Scientific, 1995; Advances in Astrofundamental Physics, Erice Chalonge Course, N. Sánchez and A. Zichichi Editors, World Scientific, 1995; D. Boyanovsky, H. J. de Vega and R. Holman, p. 183-270 in the Proceedings of the Vth. Erice School 'D. Chalonge', 'Current Topics in Astrofundamental Physics', N. Sánchez and A. Zichichi, editors, World Scientific. D. Boyanovsky, D. Cormier, H. J. de Vega, R. Holman and S. Prem Kumar, Erice Chalonge School, Current Topics in Astrofundamental Physics, N. Sánchez and A. Zichichi Editors, p. 119-194, Kluwer, 1998; D. Boyanovsky and H. J. de Vega, Out of equilibrium fields in selfconsistent inflationary dynamics: Density fluctuations astro-ph/0006446

[42] D. Boyanovsky, H. J. de Vega, R. Holman, J. F. J. Salgado, Phys. Rev. D54, 7570 (1996); D. Boyanovsky, C. Destri, H. J. de Vega, R. Holman and J. F. J. Salgado, Phys. Rev. D57, 7388 (1998); D. Boyanovsky, H. J. de Vega, R. Holman and J. F. J. Salgado, Phys. Rev. D59, 125009 (1999).

[43] J. I. Kapusta, Finite Temperature Field Theory, Cambridge Monographs on Mathematical Physics, Cambridge Univ. Press, 1989.

[44] M. Le Bellac, Thermal Field Theory, Cambridge University Press, 1996.

[45] A. Dolgov and J. Silk, Phys. Rev. D 47, 3144 (1993).

[46] I. S. Gradshteyn and I. M. Ryshik, Table of Integrals, Series and Products, Academic Press, 1980; N. N. Lebedev, Special Functions and their Applications, Prentice Hall, 1965.

[47] D. Boyanovsky, H. J. de Vega, R. Holman, S. Prem Kumar and R. D. Pisarski, Phys.Rev. D58 (1998) 125009.

[48] D. Boyanovsky, H. J. de Vega and M. Simionato, Phys. Rev. D 61, 085007 (2000). 